Journal of Environmental Sciences (JES)

Institute of Environmental Studies and Research, Ain Shams University

Elmotily, Hossam et al.

\title{
COMPARISON OF INORGANIC CHEMICAL COMPOSITIONS OF TOTAL SUSPENDED AND RESPIRABLE PARTICULATES IN DIFFERENT REGIONS OF CAIRO
}

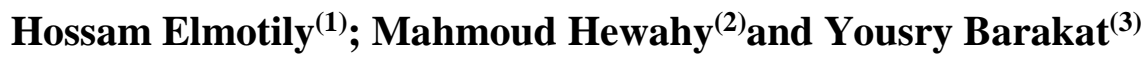 \\ 1) Central Lab for Industrial Pollution Studies, Tabbin Institute for \\ Metallurgical Studies 2) Environmental Basic Sciences Department, Institute \\ of Environmental Studies \& Research, Ain Shams University 3) Corrosion \\ and Protection of Metallic Material Department, Tabbin Institute for \\ Metallurgical Studies
}

\begin{abstract}
The inorganic chemical composition of atmospheric Total Suspended Particulate (TSP) and Particulate Matter with an aerodynadic diameter less than $10 \mu \mathrm{m}\left(\mathrm{PM}_{10}\right)$ in different regions of Cairo, in spring and autumn of 2018 was illustrated in this work. The results showed that the highest concentration of TSP and $\mathrm{PM}_{10}$ were close to industrial and heavy traffic areas "Tabbin, Helwan, Shobra Elkhimah and Fifth Settlement area".

The results showed that dust mass concentrations in Shobra Elkhimah were higher than all the other locations in both TSP and $\mathrm{PM}_{10}$. These results were pointed out in both spring and autumn.

The results showed that the concentration of heavy metals in Helwan area was higher than all other locations. These concentrations suggest that heavy metals pollution in Helwan was more serious.

Ration analysis of soluble ions showed that sodium and chloride were not from sea salt but sodium might be from the resuspension of soil dust, and chloride might be associated with the activities of burning. Potassium had
\end{abstract}

Vol.(50); Iss.(3); No.(1); Mar..2021 ISSN 1110-0826 
diversity of sources other than burning of biomass. Sulfate and nitrate were mostly from fossil fuel origin.

The $\mathrm{NO}_{3}^{-} / \mathrm{SO}_{4}^{-}$ratios in Tabbin were higher than in Helwan and Shobra Elkhimah for two types of particles during spring and autumn, indicating that the contribution of stationary emission to total particles in Tabbin were higher than in Helwan and Shobra Elkhimah.

Key words: Particulate Matter; Tabbin; Helwan; Shobra Elkhimah

\section{INTRODUCTION}

Air pollution is a process that introduces diverse pollutants into the atmosphere that cause harm to humans, other living organisms, and the natural environment. The emission of Particulate Matter (PM), may cause visible effects in form of deposition on clothes, plants, buildings and observed health effects, was noticed as the first signs of air pollution. (Kim et al., 2015, Wardencki et al, 2016).

$\mathrm{PM}$ can range in size from $0.001 \mu \mathrm{m}$ to greater than $100 \mu \mathrm{m}$ in diameter. Generally, aerosols are classified according to their aerodynamic diameter. Coarse particles are in the fraction between $10 \mu \mathrm{m}$ and $2.5 \mu \mathrm{m}$ in diameter; fine particles are between $2.5 \mu \mathrm{m}$ and $0.1 \mu \mathrm{m}$ in diameter and ultra-fine particles are less than $0.1 \mu \mathrm{m}$. PM with aerodynamic diameters less than $10 \mu \mathrm{m}$ are known as PM10 and PM with aerodynamic diameters less than $2.5 \mu \mathrm{m}$ are known as PM2.5. Total Suspended Particulate (TSP) includes all PM fractions (Pope, 2000).

Sources of PM are both natural and anthropological (Kumar, 2008). Several sources may contribute to the emission of suspended PM in the air. 
Journal of Environmental Sciences (JES)

Institute of Environmental Studies and Research, Ain Shams University

Elmotily, Hossam et al.

The main contributors are sources of urban pollution, including vehicular and industrial exhaust emissions, biomass burning, natural disasters, and other localized sources from home and occupational activities. (Peixoto et al., 2017)

Human activity affects the natural geological and biological redistribution of metals through pollution of the air, water, and soil. (Geiger and Copper, 2010).

Water-soluble ions are major components of atmospheric aerosols and can comprise up to 60-70\% of particulate mass (Ali Mohamed., 1991). Water-soluble inorganic ions (WSIIs) are a major part of fine particles $\left(\mathrm{PM}_{2.5}\right.$, Particulate Matter with an aerodynamic diameter less than $2.5 \mu \mathrm{m}$ ). Of the various components, Secondary Inorganic Ions (SII), including sulfate (SO4-), nitrate $\left(\mathrm{NO}_{3}{ }^{-}\right)$, and ammonium $\left(\mathrm{NH}_{4}{ }^{+}\right)$, are the predominant species and account for more than $90 \%$ of WSIIs (Zhang et al., 2012). Moreover, they also play important roles in atmospheric acidification and climate change (Andreae et al., 2008, Zheng et al., 2015). Sulfate is primarily formed through homogeneous gas-phase oxidation of sulfur dioxide, while heterogeneous transformation processes, i.e., metal-catalyzed oxidation, $\mathrm{H}_{2} \mathrm{O}_{2} / \mathrm{O}_{3}$ oxidation, and in-cloud process, are also reported (Seinfeld, 1986, Wang et al., 2006). Both homogeneous reaction via $\mathrm{NO}_{2}$ oxidation by $\mathrm{OH}$ radical and $\mathrm{O}_{3}$, and the heterogeneous hydrolysis of $\mathrm{N}_{2} \mathrm{O}_{5}$ on preexisting aerosols, are important pathways of nitric acid formation (Khoder, 2002). 
Journal of Environmental Sciences (JES)

Institute of Environmental Studies and Research, Ain Shams University

Elmotily, Hossam et al.

The objectives of this study are to quantify the various mass concentrations, inorganic chemical compositions and sources of TSP and $\mathrm{PM}_{10}$ in different regions of Cairo.

\section{MATERIALS AND METHODS}

The applied methodology in this study is based on US-EPA Compendium Method IO-2.1 (Sampling of TSP and PM10 Using High Volume (HV) Sampler). Concisely, the method is based on using a HighVolume Air Sampler for collection of dust particulates from ambient air onto the sampling module that consists of particle filter (i.e. glass fiber filter). This method is applicable for collecting and trapping dust particulate as well as inorganic materials present in dust and heavy metals.

This study was carried out from February to March 2018. The study areas are located in El Tabbin district with geographical coordinates of $29^{\circ} 46^{\prime} 55.60 " \mathrm{~N}$ latitude and $31^{\circ} 18^{\prime} 10.32 " \mathrm{E}$ longitude, Helwan area with geographical coordinates of $29^{\circ} 51^{\prime} 10.69^{\prime \prime} \mathrm{N}$ latitude and $31^{\circ} 20^{\prime} 16.35^{\prime \prime} \mathrm{E}$ longitude, Shobra Elkhiema area with geographical coordinates of $30^{\circ}$ 8'5.42"N latitude and $31^{\circ} 18^{\prime} 2.09 " \mathrm{E}$ longitude, Fifth settlement area with geographical coordinates of $30^{\circ} 0^{\prime} 8.61 " \mathrm{~N}$ latitude and $31^{\circ} 25^{\prime} 19.44 " \mathrm{E}$ longitude. The selected sampling area is surrounded by a mixture of urban, industrial, commercial and traffic activities. Furthermore, these areas represent a large urban industrialized area in El Tabbin district and Helwan city where metallurgical, chemical, coal, petrochemical, bricks, cement- 
producing plants are located, however in Shobra Elkhiema where Textile and Dying Plants, Lead and Cast-iron Foundries, Glass Plants, Fertilizer Plants, Soap and oil Plant, Aluminum Sulphate Plant, Petroleum Refining Plants and Gas Liquefaction Plants are located. Figure 1 shows the sampling site locations. With the aid of a global positioning system device (Garmin Global Positioning System; GPS), four sampling sites were chosen taking into consideration covering different activities.

\section{RESULTS AND DISCUSSION}

Differences in dust mass concentration: The atmospheric dust mass concentrations from different sampling sites and seasons under study are provided in Table 1. As illustrated in Table 1 and figures 2 to figure 5, the maximum value of mass concentration for TSP was $1424 \pm 8.6 \mu \mathrm{g} / \mathrm{m}^{3}$ and $1294 \pm 7.8 \mu \mathrm{g} / \mathrm{m}^{3}$ in Shobra Elkhimah and the minimum value was $394.7 \pm 79.6$ $\mu \mathrm{g} / \mathrm{m} 3$ and $439 \pm 88.4 \mu \mathrm{g} / \mathrm{m}^{3}$ in Fifth settlement at spring and autumn respectively, meanwhile the maximum value of mass concentration for PM10 was $765 \pm 29.6 \mu \mathrm{g} / \mathrm{m}^{3}$ and $695 \pm 26.9 \mu \mathrm{g} / \mathrm{m}^{3}$ in Shobra Elkhimah and the minimum value was $296 \pm 45.2 \mu \mathrm{g} / \mathrm{m}^{3}$ and $329 \pm 50.2 \mu \mathrm{g} / \mathrm{m}^{3}$ in Fifth settlement at spring and autumn respectively. 
Table(1): Statistic value for mass concentrations of TSP and PM10 at Autumn and Spring in Tabbin, Helwan, Sohbra Elkhimah and Fifth settlement.

\begin{tabular}{|c|c|c|c|c|}
\hline & & \multicolumn{2}{|c|}{ Particulate Matter } & \multirow{2}{*}{$\begin{array}{c}\text { Mass percentage (\%) } \\
\mathrm{PM}_{10} / \mathrm{TSP}\end{array}$} \\
\hline Area & $\begin{array}{c}\text { Mass } \\
\text { concentration }\end{array}$ & $\begin{array}{c}\text { TSP } \\
\left(\mu \mathrm{g} / \mathrm{m}^{3}\right)\end{array}$ & $\begin{array}{c}\mathrm{PM}_{10} \\
\left(\mu \mathrm{g} / \mathrm{m}^{3}\right)\end{array}$ & \\
\hline \multicolumn{5}{|c|}{ Tabbin } \\
\hline \multirow{5}{*}{ Autumn } & Min & 512.0 & 300.0 & 58.6 \\
\hline & $\operatorname{Max}$ & 579.0 & 374.0 & 67.3 \\
\hline & Median & 556.0 & 367.0 & 63.4 \\
\hline & Mean & 549.0 & 347.0 & 63.1 \\
\hline & SD & 34.0 & 40.9 & 4.3 \\
\hline \multirow{5}{*}{ Spring } & Min & 403.0 & 330.0 & 58.6 \\
\hline & $\operatorname{Max}$ & 639.0 & 411.0 & 102.0 \\
\hline & Median & 563.0 & 403.0 & 63.1 \\
\hline & Mean & 535.0 & 381.3 & 74.6 \\
\hline & SD & 120.5 & 44.6 & 23.9 \\
\hline \multicolumn{5}{|c|}{ Helwan } \\
\hline \multirow{5}{*}{ Autumn } & Min & 278.0 & 184.0 & 41.0 \\
\hline & Max & 788.0 & 323.0 & 66.2 \\
\hline & Median & 533.0 & 254.0 & 47.7 \\
\hline & Mean & 533.0 & 253.7 & 51.6 \\
\hline & SD & 255.0 & 69.5 & 13.1 \\
\hline \multirow{5}{*}{ Spring } & Min & 492.0 & 193.0 & 32.7 \\
\hline & $\operatorname{Max}$ & 730.0 & 516.0 & 73.3 \\
\hline & Median & 704.0 & 239.0 & 39.2 \\
\hline & Mean & 642.0 & 316.0 & 48.4 \\
\hline & SD & 130.6 & 174.7 & 21.8 \\
\hline
\end{tabular}


Cont. Table(1): Statistic value for mass concentrations of TSP and PM10 at Autumn and Spring in Tabbin, Helwan, Sohbra Elkhimah and Fifth settlement.

\begin{tabular}{|c|c|c|c|c|}
\hline & & \multicolumn{2}{|c|}{ Particulate Matter } & \multirow{2}{*}{$\begin{array}{c}\text { Mass percentage (\%) } \\
\mathrm{PM}_{10} / \mathrm{TSP}\end{array}$} \\
\hline Area & $\begin{array}{c}\text { Mass } \\
\text { concentration }\end{array}$ & $\begin{array}{c}\text { TSP } \\
\left(\mu \mathrm{g} / \mathrm{m}^{3}\right)\end{array}$ & $\begin{array}{c}\mathrm{PM}_{10} \\
\left(\mu \mathrm{g} / \mathrm{m}^{3}\right)\end{array}$ & \\
\hline \multicolumn{5}{|c|}{ Shobra ElKhimah } \\
\hline \multirow{5}{*}{ Autumn } & Min & 1286.0 & 668.0 & 51.9 \\
\hline & Max & 1302.0 & 722.0 & 55.5 \\
\hline & Median & 1294.0 & 695.0 & 53.7 \\
\hline & Mean & 1294.0 & 695.0 & 53.7 \\
\hline & $\mathrm{SD}$ & 8.0 & 27.0 & 1.8 \\
\hline \multirow{5}{*}{ Spring } & Min & 1415.0 & 34.0 & 2.4 \\
\hline & $\operatorname{Max}$ & 1423.0 & 794.0 & 55.8 \\
\hline & Median & 1423.0 & 764.0 & 53.7 \\
\hline & Mean & 1420.3 & 530.7 & 37.3 \\
\hline & SD & 4.6 & 430.4 & 30.2 \\
\hline \multicolumn{5}{|c|}{ Fifth Setlement } \\
\hline \multirow{5}{*}{ Autumn } & Min & 350.0 & 279.0 & 71.9 \\
\hline & $\operatorname{Max}$ & 527.0 & 379.0 & 79.7 \\
\hline & Median & 439.0 & 329.0 & 74.9 \\
\hline & Mean & 438.7 & 329.0 & 75.5 \\
\hline & SD & 88.5 & 50.0 & 3.9 \\
\hline \multirow{5}{*}{ Spring } & Min & 314.0 & 250.0 & 71.9 \\
\hline & Max & 474.0 & 341.0 & 79.6 \\
\hline & Median & 395.0 & 296.0 & 74.9 \\
\hline & Mean & 394.3 & 295.7 & 75.5 \\
\hline & $\mathrm{SD}$ & 80.0 & 45.5 & 3.9 \\
\hline
\end{tabular}


No significant seasonal variations of PM were observed in Tabbin, Helwan and Fifth settlement for two types of particles, but for Shobra Elkimah, TSP and PM10 were significantly different (ANOVA, p < 0.05), Meanwhile, from the linear regression analysis for TSP vs. PM10, all correlation coefficients $\left(\mathrm{r}^{2}\right)$ were higher than $0.28(\mathrm{p}<0.001)$ for four areas and the two seasons (Table 2), indicating that they have common emission sources and could be used to estimate PM10 from TSP.

Table 3 shows the averages of the mass concentrations measured as well as the mass concentrations available from some other locations of Egypt.

Table(2): Linear regression analysis for TSP vs. PM10 for the four areas and two seasons.

\begin{tabular}{|c|c|c|c|c|}
\hline Area & Season & Combination & Linear regression equation & $\mathbf{r 2}$ \\
\hline \multirow{2}{*}{ Tabbin } & Autumn & \multirow{2}{*}{ TSP vs PM10 } & PM10 = 1.0906 TSP - 251.74 & $0.826 \mathrm{a}$ \\
\hline & Spring & & PM10 = 1.0906 TSP -276.91 & 0.826 \\
\hline \multirow{2}{*}{ Helwan } & Autumn & \multirow{2}{*}{ TSP vs PM10 } & PM10 $=0.2724 \mathrm{TSP}+108.32$ & 1 \\
\hline & Spring & & PM10 $=0.7028$ TSP -135.23 & 0.2773 \\
\hline \multirow{2}{*}{$\begin{array}{c}\text { Shobra } \\
\text { Elkhimah }\end{array}$} & Autumn & \multirow{2}{*}{ TSP vs PM10 } & PM10 = 3.4289 TSP - 3742.5 & 0.9997 \\
\hline & Spring & & $\mathrm{PM} 10=3.4289 \mathrm{TSP}-4116.8$ & 0.9997 \\
\hline \multirow{2}{*}{$\begin{array}{c}\text { Fifth } \\
\text { Settlement }\end{array}$} & Autumn & \multirow{2}{*}{ TSP vs PM10 } & $\mathrm{PM} 10=0.5675 \mathrm{TSP}+80.04$ & 1 \\
\hline & Spring & & PM10 $=0.5675 \mathrm{TSP}+72.036$ & 1 \\
\hline \multicolumn{5}{|c|}{$\begin{array}{l}\text { r2: correlation coefficients. } \\
\text { a All significant level as } p<0.001 \\
\text { TSP: Total Suspended Particulate } \\
\text { Matter with an aerodynamic diameter less than } 10 \mu \mathrm{m}\end{array}$} \\
\hline
\end{tabular}


Table(3): Comparison of PM concentration $(\mu \mathrm{g} / \mathrm{m} 3)$ in studied areas and some other locations of Egypt

\begin{tabular}{|c|c|c|c|c|}
\hline Location & $\begin{array}{c}\text { Sampling } \\
\text { Time }\end{array}$ & TSP & PM10 & Reference \\
\hline \hline \multirow{2}{*}{ Tabbin } & 2018 & 576 & 364 & This study \\
\cline { 2 - 6 } & 1999 & 762.2 & --- & Borai and Soliman. (2001) \\
\hline Helwan & 2018 & 588 & 285 & This study \\
\hline $\begin{array}{c}\text { Shobra } \\
\text { Elkhimah }\end{array}$ & 2018 & 1359 & 730 & This study \\
\cline { 2 - 6 } & 1999 & 776 & --- & Borai and Soliman. (2001) \\
\hline $\begin{array}{c}\text { Fifth } \\
\text { Settlement }\end{array}$ & 2018 & 417 & 313 & This study \\
\hline Ramsis & 1999 & --- & 731.2 & Borai and Soliman. (2001) \\
\hline Nasr City & 1999 & --- & 132.8 & Borai and Soliman. (2001) \\
\hline $\begin{array}{c}\text { 10th of } \\
\text { Ramadan }\end{array}$ & 1999 & --- & 83.2 & Borai and Soliman. (2001) \\
\hline $\begin{array}{c}\text { 6th of } \\
\text { October }\end{array}$ & 1999 & --- & 97.5 & Borai and Soliman. (2001) \\
\hline \multicolumn{5}{|c|}{$\begin{array}{c}\text { TSP: Total Suspended Particulate } \\
\text { PM }\end{array}$ : Particulate Matter with an aerodynamic diameter less than 10 $\mu \mathrm{m}$} \\
\hline
\end{tabular}

- Differences in Heavy metals elements concentration: According to the results illustrated in table 4 and table 5 the measured concentrations for nine elements in TSP and $\mathrm{PM}_{10}$ in studies areas, element concentrations can be classified in to three categories

○ Elem

$\circ$ ents with high concentrations: $\mathrm{Al}, \mathrm{Mn}$, and $\mathrm{Zn}$

○ Elements with low concentrations: $\mathrm{Fe}$, and $\mathrm{Mg}$

○ Trace elements: $\mathrm{Co}, \mathrm{Cu}, \mathrm{Ni}$ and $\mathrm{Pb}$ 
Journal of Environmental Sciences (JES)

Institute of Environmental Studies and Research, Ain Shams University

Elmotily, Hossam et al.

Table(4): Statistical analysis of chemical species in TSP and PM10 at autumn from different areas of Cairo

\begin{tabular}{|c|c|c|c|c|c|c|c|c|}
\hline & \multicolumn{2}{|c|}{ Tabbin } & \multicolumn{2}{|c|}{ Helwan } & \multicolumn{2}{|c|}{ Shobra Elkhimah } & \multicolumn{2}{|c|}{ Fifth Settlement } \\
\hline & TSP & PM10 & TSP & PM10 & TSP & PM10 & TSP & PM10 \\
\hline Al & $\begin{array}{c}8.40 \pm 1.4 \\
4 \\
\end{array}$ & $\begin{array}{c}6.2580 \pm 2 \\
.3101 \\
\end{array}$ & $\begin{array}{c}37.2563 \pm \\
26.8687 \\
\end{array}$ & $\begin{array}{c}36.4535 \pm \\
28.7468 \\
\end{array}$ & $\begin{array}{c}14.8810 \pm \\
2.7978 \\
\end{array}$ & $\begin{array}{c}11.1512 \pm \\
0.0391\end{array}$ & $\begin{array}{c}10.8883 \pm \\
4.7880\end{array}$ & $\begin{array}{c}8.8548 \pm \\
3.4155 \\
\end{array}$ \\
\hline Co & $\begin{array}{c}0.008 \pm 0 . \\
001\end{array}$ & $\begin{array}{c}0.0066 \pm 0 \\
.0016 \\
\end{array}$ & $\begin{array}{c}0.0094 \pm 0 . \\
0066\end{array}$ & $\begin{array}{c}0.0168 \pm 0 . \\
0128\end{array}$ & $\begin{array}{c}0.0158 \pm 0 . \\
0057\end{array}$ & $\begin{array}{c}0.0126 \pm 0 \\
.0033 \\
\end{array}$ & $\begin{array}{c}0.0075 \pm . \\
0022\end{array}$ & $\begin{array}{c}0.0095 \pm \\
0.004 \\
\end{array}$ \\
\hline $\mathbf{C r}$ & UD & UD & UD & UD & UD & UD & UD & UD \\
\hline $\mathrm{Cu}$ & $\begin{array}{c}0.023 \pm 0 . \\
006\end{array}$ & $\begin{array}{c}0.0181 \pm 0 \\
.0058 \\
\end{array}$ & $\begin{array}{c}0.0504 \pm 0 . \\
0274\end{array}$ & $\begin{array}{c}0.0304 \pm 0 . \\
0158\end{array}$ & $\begin{array}{c}0.0482 \pm 0 . \\
0187\end{array}$ & $\begin{array}{c}0.0364 \pm 0 \\
.0035 \\
\end{array}$ & $\begin{array}{c}0.0197 \pm 0 \\
.0074 \\
\end{array}$ & $\begin{array}{c}0.0155 \pm \\
0.007 \\
\end{array}$ \\
\hline fe & $\begin{array}{c}3.2882 \pm 0 \\
.94\end{array}$ & $\begin{array}{c}1.1790 \pm 1 \\
.5134\end{array}$ & $\begin{array}{c}6.0272 \pm 3 . \\
8291\end{array}$ & $\begin{array}{c}5.2654 \pm 3 \\
7441\end{array}$ & $\begin{array}{c}7.1009 \pm 1 . \\
0421\end{array}$ & $\begin{array}{c}4.4600 \pm 0 \\
.1182\end{array}$ & $\begin{array}{c}2.7246 \pm 0 \\
.2109\end{array}$ & $\begin{array}{c}2.7551 \pm \\
0.696\end{array}$ \\
\hline $\begin{array}{c}\mathbf{M} \\
\mathbf{g}\end{array}$ & $\begin{array}{c}1.6188 \pm 0 \\
.7249 \\
\end{array}$ & $\begin{array}{c}1.5458 \pm 0 \\
.5974 \\
\end{array}$ & $\begin{array}{c}3.6644 \pm 2 . \\
2731 \\
\end{array}$ & $\begin{array}{c}1.7320 \pm 1 \\
2316 \\
\end{array}$ & $\begin{array}{c}6.5119 \pm 0 . \\
9882 \\
\end{array}$ & $\begin{array}{c}2.6608 \pm 1 \\
.2284 \\
\end{array}$ & $\begin{array}{c}5.2020 \pm 2 \\
.4825 \\
\end{array}$ & $\begin{array}{c}2.8835 \pm \\
1.718 \\
\end{array}$ \\
\hline $\begin{array}{c}\text { M } \\
\mathbf{n}\end{array}$ & $\begin{array}{c}0.5314 \pm 0 \\
.1558\end{array}$ & $\begin{array}{c}0.4476 \pm 0 \\
.2919\end{array}$ & $\begin{array}{c}25.6848 \pm \\
16.23\end{array}$ & $\begin{array}{c}17.9663 \pm \\
12.38\end{array}$ & $\begin{array}{c}0.4673 \pm 0 . \\
1220\end{array}$ & $\begin{array}{c}0.3025 \pm 0 \\
.0914\end{array}$ & $\begin{array}{c}13.2279 \pm \\
1.204\end{array}$ & $\begin{array}{c}10.3665 \\
\pm 0.69\end{array}$ \\
\hline $\mathbf{N i}$ & $\begin{array}{c}0.0221 \pm 0 \\
.0033\end{array}$ & $\begin{array}{c}0.0263 \pm 0 \\
.0143 \\
\end{array}$ & $\begin{array}{c}0.0276 \pm 0 . \\
0121\end{array}$ & $\begin{array}{c}0.0304 \pm 0 . \\
0165\end{array}$ & $\begin{array}{c}0.0373 \pm 0 . \\
0058\end{array}$ & $\begin{array}{c}0.0231 \pm 0 \\
.0061\end{array}$ & $\begin{array}{c}0.0132 \pm 0 \\
.0025\end{array}$ & $\begin{array}{c}0.0075 \pm \\
0.003 \\
\end{array}$ \\
\hline $\mathbf{P b}$ & $\begin{array}{c}0.2581 \pm 0 \\
.2512\end{array}$ & $\begin{array}{c}0.0980 \pm 0 \\
.0870\end{array}$ & $\begin{array}{c}0.0597 \pm 0 . \\
0496\end{array}$ & $\begin{array}{c}0.0098 \pm 0 . \\
0009\end{array}$ & $\begin{array}{c}0.0168 \pm 0 . \\
0049\end{array}$ & $\begin{array}{c}0.0105 \pm 0 \\
.0005\end{array}$ & $\begin{array}{c}0.0080 \pm 0 \\
.0020\end{array}$ & $\begin{array}{c}0.0057 \pm \\
0.004\end{array}$ \\
\hline Zn & $\begin{array}{c}7.7579 \pm 2 \\
.8455\end{array}$ & $\begin{array}{c}4.2841 \pm 2 \\
.3154\end{array}$ & $\begin{array}{c}47.1068 \pm \\
35.068\end{array}$ & $\begin{array}{c}43.7792 \pm \\
34.461\end{array}$ & $\begin{array}{c}5.1039 \pm 1 . \\
4962\end{array}$ & $\begin{array}{c}6.8652 \pm 1 \\
.4133\end{array}$ & $\begin{array}{c}8.2039 \pm 4 \\
.1835\end{array}$ & $\begin{array}{c}7.1112 \pm \\
3.273\end{array}$ \\
\hline $\mathrm{SO}_{a}^{z-}$ & $\begin{array}{c}16.5842 \pm \\
1.755 \\
\end{array}$ & $\begin{array}{c}12.7377 \pm \\
1.038 \\
\end{array}$ & $\begin{array}{c}54.7543 \pm \\
44.592 \\
\end{array}$ & $\begin{array}{c}37.5612 \pm \\
30.348 \\
\end{array}$ & $\begin{array}{c}21.7575 \pm \\
1.7675 \\
\end{array}$ & $\begin{array}{c}16.9516 \pm \\
3.332 \\
\end{array}$ & $\begin{array}{c}14.3465 \pm \\
4.184 \\
\end{array}$ & $\begin{array}{c}12.9488 \\
\pm 3.658 \\
\end{array}$ \\
\hline $\mathrm{NO}_{\mathrm{n}}^{-}$ & $\begin{array}{c}19.1418 \pm \\
6.489 \\
\end{array}$ & $\begin{array}{c}11.6432 \pm \\
5.749 \\
\end{array}$ & $\begin{array}{c}22.8847 \pm \\
13.6369 \\
\end{array}$ & $\begin{array}{c}35.1403 \pm \\
27.102 \\
\end{array}$ & $\begin{array}{c}7.9971 \pm 2 . \\
0755\end{array}$ & $\begin{array}{c}9.5924 \pm 0 \\
.4350 \\
\end{array}$ & $\begin{array}{c}10.1218 \pm \\
6.0786 \\
\end{array}$ & $\begin{array}{c}9.1986 \pm \\
5.3126 \\
\end{array}$ \\
\hline$F^{-}$ & $\begin{array}{c}0.2253 \pm 0 \\
.1490\end{array}$ & $\begin{array}{c}0.1279 \pm 0 \\
.0119\end{array}$ & $\begin{array}{c}0.6328 \pm 0 \\
4983\end{array}$ & $\begin{array}{c}0.8181 \pm 0 . \\
7260\end{array}$ & $\begin{array}{c}0.4974 \pm 0 . \\
2505\end{array}$ & $\begin{array}{c}0.4675 \pm 0 \\
.1059\end{array}$ & $\begin{array}{c}0.3528 \pm 0 \\
.2573\end{array}$ & $\begin{array}{c}0.3025 \pm \\
0.1976 \\
\end{array}$ \\
\hline $\mathrm{cl}^{-}$ & $\begin{array}{c}35.2605 \pm \\
8.779 \\
\end{array}$ & $\begin{array}{c}27.5700 \pm \\
9.965 \\
\end{array}$ & $\begin{array}{c}34.0770 \pm \\
27.685 \\
\end{array}$ & $\begin{array}{c}33.6836 \pm \\
29.158 \\
\end{array}$ & $\begin{array}{c}51.7647 \pm \\
36.012 \\
\end{array}$ & $\begin{array}{c}13.8132 \pm \\
1.766 \\
\end{array}$ & $\begin{array}{c}12.8465 \pm \\
1.855 \\
\end{array}$ & $\begin{array}{r}13.4674 \\
\pm 0.799 \\
\end{array}$ \\
\hline $\mathrm{NH}_{2}^{+}$ & $\begin{array}{c}2.0672 \pm 0 \\
.9220\end{array}$ & $\begin{array}{c}2.6526 \pm 0 \\
.9130\end{array}$ & UD & UD & UD & UD & UD & UD \\
\hline $\mathrm{Na}^{+}$ & $\begin{array}{c}4.4645 \pm 3 \\
.2127\end{array}$ & $\begin{array}{c}2.3807 \pm 1 \\
.0549\end{array}$ & $\begin{array}{c}2.9499 \pm 0 . \\
9095\end{array}$ & $\begin{array}{c}5.5406 \pm 4 . \\
2395\end{array}$ & $\begin{array}{c}22.0250 \pm \\
17.4178\end{array}$ & $\begin{array}{c}3.3585 \pm 0 \\
.5805\end{array}$ & UD & UD \\
\hline $\mathbf{K}^{+}$ & $\begin{array}{c}1.4713 \pm 0 \\
.4104\end{array}$ & $\begin{array}{c}1.1149 \pm 0 \\
.3164\end{array}$ & $\begin{array}{c}8.1166 \pm 6 . \\
8459\end{array}$ & $\begin{array}{c}8.0539 \pm 6 . \\
8829\end{array}$ & $\begin{array}{c}2.2835 \pm 0 \\
2799\end{array}$ & $\begin{array}{c}1.6430 \pm 0 \\
.1206\end{array}$ & $\begin{array}{c}0.8379 \pm 0 \\
.3455\end{array}$ & $\begin{array}{c}0.8897 \pm \\
0.2913\end{array}$ \\
\hline $\mathrm{Ca}^{2+}$ & $\begin{array}{c}22.3532 \pm \\
7.6761\end{array}$ & $\begin{array}{c}13.9105 \pm \\
1.5283\end{array}$ & $\begin{array}{c}31.8734 \pm \\
26.6703 \\
\end{array}$ & $\begin{array}{c}19.2736 \pm \\
15.4603 \\
\end{array}$ & $\begin{array}{c}19.5824 \pm \\
7.7858 \\
\end{array}$ & $\begin{array}{c}10.9560 \pm \\
1.9442\end{array}$ & $\begin{array}{c}6.5161 \pm 3 \\
.1196\end{array}$ & $\begin{array}{c}.9522 \pm \\
0.0287\end{array}$ \\
\hline $\mathrm{Mg}^{\mathrm{2}}$ & $\begin{array}{c}1.2115 \pm 0 \\
.1785\end{array}$ & $\begin{array}{c}0.7820 \pm 0 \\
.1791\end{array}$ & $\begin{array}{c}3.7953 \pm 3 . \\
2110\end{array}$ & $\begin{array}{c}3.0715 \pm 2 . \\
4409\end{array}$ & $\begin{array}{c}2.9695 \pm 1 \\
5444\end{array}$ & $\begin{array}{c}1.1228 \pm 0 \\
.0217\end{array}$ & $\begin{array}{c}0.22450 \\
1290\end{array}$ & $\begin{array}{c}0.3803 \pm \\
0.2793\end{array}$ \\
\hline
\end{tabular}


Journal of Environmental Sciences (JES)

Institute of Environmental Studies and Research, Ain Shams University

Elmotily, Hossam et al.

Table(5): Statistical analysis of chemical species in TSP and PM10 at spring from different areas of Cairo

\begin{tabular}{|c|c|c|c|c|c|c|c|c|}
\hline & \multicolumn{2}{|c|}{ Tabbin } & \multicolumn{2}{|c|}{ Helwan } & \multicolumn{2}{|c|}{ Shobra Elkhimah } & \multicolumn{2}{|c|}{ Fifth Settlement } \\
\hline & TSP & PM10 & TSP & PM10 & TSP & PM10 & TSP & PM10 \\
\hline Al & $\begin{array}{c}10.1693 \pm \\
1.7468\end{array}$ & $\begin{array}{c}7.2459 \pm 2 . \\
2374\end{array}$ & $\begin{array}{c}33.5854 \pm \\
1.0819\end{array}$ & $\begin{array}{c}11.4819 \pm \\
2.9853\end{array}$ & $\begin{array}{c}18.0101 \pm \\
3.3884\end{array}$ & $\begin{array}{c}13.4938 \pm \\
0.0447\end{array}$ & $\begin{array}{c}8.8165 \pm 3 \\
.8761\end{array}$ & $\begin{array}{c}7.1712 \pm 2 \\
.7698\end{array}$ \\
\hline Co & $\begin{array}{c}0.0093 \pm 0 . \\
0012\end{array}$ & $\begin{array}{c}0.0077 \pm 0 . \\
0013\end{array}$ & $\begin{array}{c}0.0087 \pm 0 . \\
0000\end{array}$ & $\begin{array}{c}0.0055 \pm 0 \\
.0011\end{array}$ & $\begin{array}{c}0.0191 \pm 0 . \\
0069\end{array}$ & $\begin{array}{c}0.0152 \pm 0 \\
.0040\end{array}$ & $\begin{array}{c}0.0060 \pm 0 \\
.0018\end{array}$ & $\begin{array}{c}0.0077 \pm 0 \\
.0030\end{array}$ \\
\hline $\mathrm{Cr}$ & UD & UD & UD & UD & UD & UD & UD & UD \\
\hline $\mathrm{Cu}$ & $\begin{array}{c}0.0284 \pm 0 . \\
0077\end{array}$ & $\begin{array}{c}0.0210 \pm 0 . \\
0057\end{array}$ & $\begin{array}{c}0.0570 \pm 0 . \\
0150\end{array}$ & $\begin{array}{c}0.0132 \pm 0 \\
.0029\end{array}$ & $\begin{array}{c}0.0583 \pm 0 . \\
0226\end{array}$ & $\begin{array}{c}0.0441 \pm 0 \\
.0042\end{array}$ & $\begin{array}{c}0.0160 \pm 0 \\
.0060\end{array}$ & $\begin{array}{c}0.0126 \pm 0 \\
.0053\end{array}$ \\
\hline fe & $\begin{array}{c}3.9787 \pm 1 . \\
1425\end{array}$ & $\begin{array}{c}2.1574 \pm 1 \\
6676\end{array}$ & $\begin{array}{c}6.1032 \pm 0 . \\
7747\end{array}$ & $\begin{array}{c}1.8382 \pm 0 \\
.1609\end{array}$ & $\begin{array}{c}8.5940 \pm 1 . \\
2623\end{array}$ & $\begin{array}{c}5.3970 \pm 0 \\
.1440 \\
\end{array}$ & $\begin{array}{c}2.2062 \pm 0 \\
.1705 \\
\end{array}$ & $\begin{array}{c}2.2311 \pm 0 \\
.5648 \\
\end{array}$ \\
\hline $\begin{array}{c}\mathbf{M} \\
\mathrm{g} \\
\end{array}$ & $\begin{array}{c}1.9588 \pm 0 . \\
8771 \\
\end{array}$ & $\begin{array}{c}1.7921 \pm 0 \\
6110 \\
\end{array}$ & $\begin{array}{c}3.7815 \pm 0 . \\
5716 \\
\end{array}$ & $\begin{array}{c}0.6047 \pm 0 \\
.0529 \\
\end{array}$ & $\begin{array}{c}7.8812 \pm 1 \\
1970 \\
\end{array}$ & $\begin{array}{c}3.2201 \pm 1 \\
.4871 \\
\end{array}$ & $\begin{array}{c}4.2121 \pm 2 \\
.0097 \\
\end{array}$ & $\begin{array}{c}2.8878 \pm 1 \\
.9446 \\
\end{array}$ \\
\hline $\begin{array}{c}\mathrm{M} \\
\mathbf{n}\end{array}$ & $\begin{array}{c}0.6430 \pm 0 \\
1885\end{array}$ & $\begin{array}{c}0.5131 \pm 0 . \\
3051\end{array}$ & $\begin{array}{c}26.1173 \pm \\
3.4552\end{array}$ & $\begin{array}{c}6.4456 \pm 0 \\
.2883 \\
\end{array}$ & $\begin{array}{c}0.5655 \pm 0 . \\
1478\end{array}$ & $\begin{array}{c}0.3660 \pm 0 \\
.1105\end{array}$ & $\begin{array}{c}10.7113 \pm \\
0.9733\end{array}$ & $\begin{array}{c}8.3937 \pm 0 \\
.5656\end{array}$ \\
\hline $\mathbf{N i}$ & $\begin{array}{c}0.0267 \pm 0 . \\
0040\end{array}$ & $\begin{array}{c}0.0303 \pm 0 . \\
0147\end{array}$ & $\begin{array}{c}0.0350 \pm 0 . \\
0135\end{array}$ & $\begin{array}{c}0.0129 \pm 0 \\
.0025 \\
\end{array}$ & $\begin{array}{c}0.0451 \pm 0 . \\
0070\end{array}$ & $\begin{array}{c}0.0280 \pm 0 \\
.0074 \\
\end{array}$ & $\begin{array}{c}0.0107 \pm 0 \\
.0020 \\
\end{array}$ & $\begin{array}{c}0.0061 \pm 0 \\
.0023 \\
\end{array}$ \\
\hline $\mathbf{P b}$ & $\begin{array}{c}0.3123 \pm 0 \\
3039 \\
\end{array}$ & $\begin{array}{c}0.1884 \pm 0 . \\
1929 \\
\end{array}$ & $\begin{array}{c}0.0453 \pm 0 . \\
0137\end{array}$ & $\begin{array}{c}0.0061 \pm 0 \\
.0038 \\
\end{array}$ & $\begin{array}{c}0.0204 \pm 0 . \\
0060\end{array}$ & $\begin{array}{c}0.0127 \pm 0 \\
.0006 \\
\end{array}$ & $\begin{array}{c}0.0065 \pm 0 \\
.0017 \\
\end{array}$ & $\begin{array}{c}0.0046 \pm 0 \\
.0035 \\
\end{array}$ \\
\hline Zn & $\begin{array}{c}9.3871 \pm 3 \\
4431\end{array}$ & $\begin{array}{c}4.9287 \pm 2 . \\
3602\end{array}$ & $\begin{array}{c}41.0474 \pm \\
3.3781\end{array}$ & $\begin{array}{c}13.8169 \pm \\
3.5438\end{array}$ & $\begin{array}{c}6.1772 \pm 1 . \\
8116\end{array}$ & $\begin{array}{c}8.3078 \pm 1 \\
.7118 \\
\end{array}$ & $\begin{array}{c}6.6428 \pm 3 \\
.3868 \\
\end{array}$ & $\begin{array}{c}5.7593 \pm 2 \\
.6532\end{array}$ \\
\hline $\mathrm{SO}_{\mathrm{a}}^{\mathrm{z}-}$ & $\begin{array}{c}32.2697 \pm \\
3.4156\end{array}$ & $\begin{array}{c}23.9689 \pm \\
0.8949\end{array}$ & $\begin{array}{c}42.7535 \pm \\
10.9551\end{array}$ & $\begin{array}{c}11.5105 \pm \\
3.5578\end{array}$ & $\begin{array}{c}26.3316 \pm \\
2.1357\end{array}$ & $\begin{array}{c}20.5120 \pm \\
4.0274\end{array}$ & $\begin{array}{c}11.6168 \pm \\
3.3865\end{array}$ & $\begin{array}{c}10.4860 \pm \\
2.9678\end{array}$ \\
\hline $\mathrm{NO}_{\mathrm{n}}^{-}$ & $\begin{array}{c}37.2464 \pm \\
12.6279 \\
\end{array}$ & $\begin{array}{c}22.2253 \pm \\
11.6898 \\
\end{array}$ & $\begin{array}{c}22.3539 \pm \\
3.3018 \\
\end{array}$ & $\begin{array}{c}10.6824 \pm \\
2.2409 \\
\end{array}$ & $\begin{array}{c}9.6788 \pm 2 . \\
5132 \\
\end{array}$ & $\begin{array}{c}11.6075 \pm \\
0.5241 \\
\end{array}$ & $\begin{array}{c}8.1956 \pm 4 \\
.9213 \\
\end{array}$ & $\begin{array}{c}7.4505 \pm 4 \\
.3060 \\
\end{array}$ \\
\hline$F^{-}$ & $\begin{array}{c}0.4384 \pm 0 \\
2900\end{array}$ & $\begin{array}{c}0.2409 \pm 0 . \\
0189\end{array}$ & $\begin{array}{c}0.5161 \pm 0 . \\
0953\end{array}$ & $\begin{array}{c}0.2221 \pm 0 \\
.1205\end{array}$ & $\begin{array}{c}0.6020 \pm 0 . \\
3033\end{array}$ & $\begin{array}{c}0.5658 \pm 0 \\
.1282 \\
\end{array}$ & $\begin{array}{c}0.2857 \pm 0 \\
.2083 \\
\end{array}$ & $\begin{array}{c}0.2450 \pm 0 \\
.1601 \\
\end{array}$ \\
\hline $\mathrm{Cl}^{-}$ & $\begin{array}{c}68.6103 \pm \\
17.0830 \\
\end{array}$ & $\begin{array}{c}51.6311 \pm \\
17.6218 \\
\end{array}$ & $\begin{array}{c}26.6955 \pm \\
6.6942 \\
\end{array}$ & $\begin{array}{c}9.4668 \pm 4 \\
.4770 \\
\end{array}$ & $\begin{array}{c}62.6423 \pm \\
43.5755 \\
\end{array}$ & $\begin{array}{c}16.7147 \pm \\
2.1331 \\
\end{array}$ & $\begin{array}{c}10.4028 \pm \\
1.5031 \\
\end{array}$ & $\begin{array}{c}8.1432 \pm 2 \\
.1070 \\
\end{array}$ \\
\hline $\mathrm{NH}_{2}^{+}$ & $\begin{array}{c}4.0223 \pm 1 . \\
7941\end{array}$ & $\begin{array}{c}5.0673 \pm 1 . \\
9397\end{array}$ & UD & UD & UD & UD & UD & UD \\
\hline $\mathrm{Na}^{+}$ & $\begin{array}{c}8.6871 \pm 6 . \\
2514\end{array}$ & $\begin{array}{c}4.4910 \pm 2 . \\
0638\end{array}$ & $\begin{array}{c}4.2355 \pm 2 . \\
1491\end{array}$ & $\begin{array}{c}1.8023 \pm 0 \\
.3678 \\
\end{array}$ & $\begin{array}{c}26.6529 \pm \\
21.0763 \\
\end{array}$ & $\begin{array}{c}4.0639 \pm 0 \\
.7016 \\
\end{array}$ & UD & UD \\
\hline $\mathbf{K}^{+}$ & $\begin{array}{c}2.8629 \pm 0 . \\
7986\end{array}$ & $\begin{array}{c}2.0828 \pm 0 \\
4718\end{array}$ & $\begin{array}{c}6.0325 \pm 2 . \\
0566\end{array}$ & $\begin{array}{c}2.3027 \pm 1 \\
.0116\end{array}$ & $\begin{array}{c}2.7635 \pm 0 \\
3384\end{array}$ & $\begin{array}{c}1.9881 \pm 0 \\
.1456\end{array}$ & $\begin{array}{c}0.6785 \pm 0 \\
.2797\end{array}$ & $\begin{array}{c}0.7205 \pm 0 \\
.2363\end{array}$ \\
\hline $\mathrm{Ca}^{2+}$ & $\begin{array}{c}43.4952 \pm \\
14.9362 \\
\end{array}$ & $\begin{array}{c}26.1887 \pm \\
2.4692 \\
\end{array}$ & $\begin{array}{c}23.9654 \pm \\
7.6846 \\
\end{array}$ & $\begin{array}{c}5.9556 \pm 1 \\
.7515 \\
\end{array}$ & $\begin{array}{c}23.6983 \pm \\
9.4197 \\
\end{array}$ & $\begin{array}{c}13.2572 \pm \\
2.3500 \\
\end{array}$ & $\begin{array}{c}5.2768 \pm 2 \\
.5267 \\
\end{array}$ & $\begin{array}{c}4.0096 \pm 0 \\
.0208 \\
\end{array}$ \\
\hline $\mathrm{Mg}^{2+}$ & $\begin{array}{c}2.3573 \pm 0 \\
3473 \\
\end{array}$ & $\begin{array}{c}1.4668 \pm 0 . \\
2888\end{array}$ & $\begin{array}{c}2.8080 \pm 0 . \\
9797\end{array}$ & $\begin{array}{c}0.9592 \pm 0 \\
.2640 \\
\end{array}$ & $\begin{array}{c}3.5935 \pm 1 . \\
8687\end{array}$ & $\begin{array}{c}1.3587 \pm 0 \\
.0265 \\
\end{array}$ & $\begin{array}{c}0.1818 \pm 0 \\
.1044 \\
\end{array}$ & $\begin{array}{c}0.3080 \pm 0 \\
.226 \\
\end{array}$ \\
\hline
\end{tabular}

○ Heavy metals Enrichment Factors (EF): Enrichment Factors (EF) were assigned for different elements to distinguish between natural and anthropogenic sources. Therefore, its use helps to determine whether a certain element has additional or anthropogenic sources other than its

Vol.(50); Iss.(3); No.(1); Mar..2021 
Journal of Environmental Sciences (JES)

Institute of Environmental Studies and Research, Ain Shams University

Elmotily, Hossam et al.

major natural sources. The EF method normalizes the measured elemental content with respect to a sample reference element. $\mathrm{Al}, \mathrm{Si}, \mathrm{Ti}$ and $\mathrm{Fe}$ are commonly used as reference elements for the main source of the Earth's crust composition.

For this study, Al was used as a reference because it is a major constituent of clay minerals and has been successfully used by several researchers as tracer.

The EFs of the selected element detected in the four areas were calculated using the following equation: $\mathrm{EFX}=(\mathrm{Cx} / \mathrm{Cref}) \mathrm{PM} /(\mathrm{Cx} / \mathrm{Cref})$ crust

where EFx is the enrichment factor of species $\mathrm{x}$ that represents the chemical element of interest. Cx represents the concentration of the element of interest and Cref is the concentration of a reference element. (Cx/Cref) PM is the concentration ratio of element $\mathrm{x}$ to the reference element in the aerosol sample and $(\mathrm{Cx} / \mathrm{Cref})$ crust is the concentration ratio of $\mathrm{x}$ to the reference element in the upper continental crust. For analysis, elements with EF value close to 1, are considered as crustal or as natural, $\mathrm{EF}<10$ were considered as non-enriched, $10<\mathrm{EF}<100$ as moderately enriched and EF $>100$ as highly enriched (Silva et al., 2019).

From figure 6 to figure 13 represent the current elements concentrations and enrichment factors of elements in Particulate Matter from different areas in Cairo at autumn and spring seasons. 
- Heavy metals statistical analysis: Table 6 represent bivariate Pearson correlations between heavy metals in Particulate Matter from different areas in Cairo at autumn and spring seasons.

Table(6): Bivariate Pearson correlations between heavy metals in TSP and PM10

\begin{tabular}{|c|c|c|c|c|c|c|c|c|c|}
\hline \multicolumn{10}{|c|}{ Tabbin } \\
\hline & Al & Co & $\mathbf{C u}$ & $\mathbf{F e}$ & Mg & Mn & $\mathrm{Ni}$ & $\mathbf{P b}$ & Zn \\
\hline \multicolumn{10}{|c|}{ TSP } \\
\hline Al & 1 & & & & & & & & \\
\hline Co & $\begin{array}{l}-.008 \\
\end{array}$ & 1 & & & & & & & \\
\hline $\mathbf{C u}$ & $.829 *$ & .246 & 1 & & & & & & \\
\hline $\mathrm{Fe}$ & .471 & -.405 & -.102 & 1 & & & & & \\
\hline $\mathrm{Mg}$ & $.878 *$ & -.021 & $.963 * *$ & .044 & 1 & & & & \\
\hline Mn & .057 & -.205 & -.491 & $.876^{*}$ & -.414 & 1 & & & \\
\hline $\mathrm{Ni}$ & .707 & -.178 & .201 & $.941 * *$ & .295 & .746 & 1 & & \\
\hline $\mathbf{P b}$ & .083 & -.511 & -.486 & $.916 *$ & -.329 & $.946 * *$ & .734 & 1 & \\
\hline $\mathbf{Z i}$ & $.959 * *$ & -.242 & $.816 *$ & .420 & $.922 * *$ & -.054 & .608 & .062 & 1 \\
\hline \multicolumn{10}{|c|}{ PM10 } \\
\hline Al & 1 & & & & & & & & \\
\hline Co & $.975 * *$ & 1 & & & & & & & \\
\hline $\mathbf{C u}$ & $.939 * *$ & $.880 *$ & 1 & & & & & & \\
\hline $\mathbf{F e}$ & $.864 *$ & .755 & $.969 * *$ & 1 & & & & & \\
\hline Mg & .736 & $.819 *$ & .466 & .300 & 1 & & & & \\
\hline Mn & $.989 * *$ & $.932 * *$ & $.944 * *$ & $.905 *$ & .674 & 1 & & & \\
\hline $\mathbf{N i}$ & $.898 *$ & $.922 * *$ & .690 & .571 & $.951 * *$ & $.866 *$ & 1 & & \\
\hline $\mathbf{P b}$ & $.978 * *$ & $.916 *$ & $.911 *$ & $.878 *$ & .699 & $.996 * *$ & $.886^{*}$ & 1 & \\
\hline $\mathbf{Z i}$ & $.977 * *$ & $.963 * *$ & $.845^{*}$ & .760 & $.845 *$ & $.964 * *$ & $.968 * *$ & $.972 * *$ & 1 \\
\hline
\end{tabular}


Journal of Environmental Sciences (JES)

Institute of Environmental Studies and Research, Ain Shams University

Elmotily, Hossam et al.

Cont. Table(6): Bivariate Pearson correlations between heavy metals in TSP and PM10

\begin{tabular}{|c|c|c|c|c|c|c|c|c|c|}
\hline \multicolumn{10}{|c|}{ Helwan } \\
\hline & Al & Co & $\mathrm{Cu}$ & Fe & $\mathrm{Mg}$ & Mn & $\mathbf{N i}$ & $\mathbf{P b}$ & $\mathbf{Z n}$ \\
\hline \multicolumn{10}{|c|}{ TSP } \\
\hline Al & 1 & & & & & & & & \\
\hline Co & $.999 * *$ & 1 & & & & & & & \\
\hline $\mathbf{C u}$ & $.816^{*}$ & $.844 *$ & 1 & & & & & & \\
\hline $\mathbf{F e}$ & $.963 * *$ & $.975 * *$ & $.942 * *$ & 1 & & & & & \\
\hline Mg & $947 * *$ & $.961 * *$ & $.959 \% *$ & $.999 * *$ & 1 & & & & \\
\hline Mn & $.959 * *$ & $.972 * *$ & $.946 * \%$ & $1.000 * *$ & $.999 * *$ & 1 & & & \\
\hline $\mathrm{Ni}$ & .560 & .600 & $.936 * *$ & .764 & .797 & .771 & 1 & & \\
\hline $\mathbf{P b}$ & $.968^{* * *}$ & $.954 * *$ & .644 & $.863 *$ & $.834 *$ & $.857 *$ & .332 & 1 & \\
\hline $\mathbf{Z i}$ & $.998 * *$ & $.994 * *$ & .778 & $.944 * *$ & .924** & $.940 * *$ & .507 & $.982^{* * *}$ & 1 \\
\hline \multicolumn{10}{|c|}{ PM10 } \\
\hline Al & 1 & & & & & & & & \\
\hline Co & $1.000 * *$ & 1 & & & & & & & \\
\hline $\mathbf{C u}$ & $.970 * *$ & $.975 * *$ & 1 & & & & & & \\
\hline $\mathbf{F e}$ & $.998 * *$ & $.999 * *$ & $.982 * *$ & 1 & & & & & \\
\hline Mg & $.998 * *$ & $.999 * *$ & $.982 * *$ & $1.000 * *$ & 1 & & & & \\
\hline Mn & $.997 * *$ & $.998 * *$ & $.985 * *$ & $1.000 * *$ & $1.000 * *$ & 1 & & & \\
\hline $\mathrm{Ni}$ & $.977 * *$ & $.980 * *$ & $1.000 * *$ & $.987 * *$ & $.987 * *$ & $.990 * *$ & 1 & & \\
\hline $\mathbf{P b}$ & .457 & .473 & .658 & .505 & .505 & .520 & .638 & 1 & \\
\hline $\mathbf{Z i}$ & $1.000 * *$ & $1.000 * *$ & $.971 * *$ & $.999 * *$ & $.999 * *$ & $.997 * *$ & $.977 * *$ & .458 & 1 \\
\hline \multicolumn{10}{|c|}{ Shobra Elkhimah } \\
\hline & Al & Co & $\mathrm{Cu}$ & $\mathbf{F e}$ & Mg & Mn & $\mathrm{Ni}$ & $\mathbf{P b}$ & $\mathbf{Z n}$ \\
\hline \multicolumn{10}{|c|}{ TSP } \\
\hline Al & 1 & & & & & & & & \\
\hline Co & $.971 * *$ & 1 & & & & & & & \\
\hline $\mathbf{C u}$ & $.966 * *$ & $1.000 * *$ & 1 & & & & & & \\
\hline $\mathbf{F e}$ & $.993 * *$ & $.937 * *$ & $.930 * *$ & 1 & & & & & \\
\hline Mg & $.995 * *$ & $.943 * *$ & $.935 * *$ & $1.000 * *$ & 1 & & & & \\
\hline Mn & $.991 * *$ & $.994 * *$ & $.992 * *$ & $.969 * *$ & $.973 * *$ & 1 & & & \\
\hline $\mathbf{N i}$ & $.996 * *$ & $.946^{* * *}$ & $.939 * *$ & $1.000 * *$ & $1.000 * *$ & $.975 * *$ & 1 & & \\
\hline $\mathbf{P b}$ & $.985 * *$ & $.998 * *$ & $.996 * *$ & $.958 * *$ & $.963 * *$ & $.999 * *$ & $.965 * *$ & 1 & \\
\hline $\mathbf{Z i}$ & $.985 * *$ & $.998 * *$ & $.996 * *$ & $.958 * *$ & $.962 * *$ & $.999 * *$ & $.965 * *$ & $1.000 * *$ & 1 \\
\hline \multicolumn{10}{|c|}{ PM10 } \\
\hline Al & 1 & & & & & & & & \\
\hline Co & .373 & 1 & & & & & & & \\
\hline $\mathbf{C u}$ & .753 & .891* & 1 & & & & & & \\
\hline $\mathbf{F e}$ & $.968 * *$ & .595 & $.894 *$ & 1 & & & & & \\
\hline Mg & .215 & $.986 * *$ & .805 & .454 & 1 & & & & \\
\hline Mn & .385 & -.712 & -.317 & .140 & $-.818^{*}$ & 1 & & & \\
\hline $\mathrm{Ni}$ & .427 & -.680 & -.274 & .185 & -.791 & $.999 * *$ & 1 & & \\
\hline $\mathbf{P b}$ & $.945 * *$ & .049 & .496 & $.832 *$ & -.116 & .666 & .699 & 1 & \\
\hline $\mathbf{Z i}$ & .465 & $.995 * *$ & $.933 * *$ & .673 & $.965 * *$ & -.638 & -.602 & .150 & 1 \\
\hline
\end{tabular}


Cont. Table(6): Bivariate Pearson correlations between heavy metals in TSP and PM10

\begin{tabular}{|c|c|c|c|c|c|c|c|c|c|}
\hline \multicolumn{10}{|c|}{ Fifth Settlement } \\
\hline & Al & Co & $\mathbf{C u}$ & $\mathbf{F e}$ & Mg & Mn & $\mathrm{Ni}$ & $\mathbf{P b}$ & $\mathbf{Z n}$ \\
\hline \multicolumn{10}{|c|}{ TSP } \\
\hline Al & 1 & & & & & & & & \\
\hline Co & $.993 * *$ & 1 & & & & & & & \\
\hline $\mathbf{C u}$ & $.999 * *$ & $.997 * *$ & 1 & & & & & & \\
\hline $\mathrm{Fe}$ & .736 & $.812 *$ & .767 & 1 & & & & & \\
\hline Mg & $1.000 * *$ & $.990 * *$ & $.998 * *$ & .721 & 1 & & & & \\
\hline Mn & .784 & $.853 *$ & $.812 *$ & $.997 * *$ & .771 & 1 & & & \\
\hline $\mathrm{Ni}$ & $.953 * *$ & $.982 * *$ & $.966 * *$ & $.907 *$ & $.946 * *$ & $.935 * *$ & 1 & & \\
\hline $\mathbf{P b}$ & -.731 & -.644 & -.698 & -.076 & -.746 & -.150 & -.490 & 1 & \\
\hline $\mathbf{Z i}$ & $.999 * *$ & $.988 * *$ & $.996 * *$ & .710 & $1.000 * *$ & .760 & $.941 * *$ & -.756 & 1 \\
\hline \multicolumn{10}{|c|}{ PM10 } \\
\hline Al & 1 & & & & & & & & \\
\hline Co & $1.000 * *$ & 1 & & & & & & & \\
\hline $\mathbf{C u}$ & $1.000 * *$ & $1.000 * *$ & 1 & & & & & & \\
\hline $\mathbf{F e}$ & $.993 * *$ & $.993 * *$ & $.990 * *$ & 1 & & & & & \\
\hline Mg & $.968 * *$ & $.967 * *$ & $.972 * *$ & $.936 * *$ & 1 & & & & \\
\hline Mn & .804 & .806 & .791 & $.870^{*}$ & .656 & 1 & & & \\
\hline $\mathrm{Ni}$ & $-.840 *$ & $-.839 *$ & $-.852 *$ & -.769 & $-.925 * *$ & -.353 & 1 & & \\
\hline $\mathbf{P b}$ & $-.911 *$ & $-.910 *$ & $-.920 * *$ & $-.855^{*}$ & $-.967 * *$ & -.488 & $.989 * *$ & 1 & \\
\hline $\mathbf{Z i}$ & $.999 * *$ & $.999 * *$ & $1.000 * *$ & $.987 * *$ & $.975 * *$ & .780 & $-.861 *$ & $-.927 * *$ & 1 \\
\hline \multirow{2}{*}{\multicolumn{10}{|c|}{ *. Correlation is significant at the 0.05 level (2-tailed). }} \\
\hline & & & & & & & & & \\
\hline
\end{tabular}

Differences in mass concentrations of water-soluble ions: Water

soluble anions (sulfate, nitrate, fluoride and chloride) and cations (sodium, potassium, calcium, ammonium and magnesium) were determined with ion chromatography at TSP and PM10 in four regions (Tabbin, Helwan, Shobra Elkhimah and Fifth Settlement). In Autumn, the mass of total WSI contributed about 19\%, 30\% of the total TSP mass in Tabbin and Helwan, and $10 \%$ in Shobra Elkhimah and Fifth Settlement, meanwhile it contributed about $21 \%, 56 \%, 8 \%$ and $10 \%$ of the PM10 mass in Tabbin, Helwan, Shobra 
Journal of Environmental Sciences (JES)

Institute of Environmental Studies and Research, Ain Shams University

Elmotily, Hossam et al.

Elkhimah and Fifth Settlement, respectively as shown in table 4. While in Spring, the mass of total WSI contributed about 33\%, 20\%, $11 \%$ and $9 \%$ of the total TSP mass in Tabbin, Helwan, Shobra Elkhimah and Fifth Settlement, respectively, meanwhile it contributed about 36\%, 14\%, 9\% and $12 \%$ of the PM10 mass in Tabbin, Helwan, Shobra Elkhimah and Fifth Settlement, respectively as shown in table 5 .

At Autumn and Spring, chloride, calcium, nitrate and sulfate are the main constituents in water soluble ionic species at TSP and PM10 in Tabbin, Helwan and Fifth Settlement while in Shobra Elkhimah, chloride, sodium, calcium and sulphate at TSP and chloride, calcium, nitrate and sulfate at PM10 are the main constituents.

\section{- Differences in specific ionic ratios}

\section{○ Enrichment Factors (EF)}

The suspended particles in air commonly hold marine and non-marine sources. The non-marine source hold both anthropogenic as well as crustal form. To detect the marine source, the soluble components compared with the sea water composition. To inspect the conceivable sources of the chemical components (marine, crustal or anthropogenic), the enrichment factor (EF) as defined below is used:

$$
\mathbf{E F}(\mathrm{X})=(\mathrm{X} / \mathrm{Me})_{\mathrm{aer}} /(\mathrm{X} / \mathrm{Me})
$$

where $(\mathrm{X} / \mathrm{Me})$ aer is the ratio of any element $\mathrm{X}$ with respect to the reference element $\mathrm{Me}$ in the aerosol and $(\mathrm{X} / \mathrm{Me})$ is the conformable ratio in the seawater. For seawater, Me is constantly taken as $\mathrm{Na}+$. Enrichment factor 
is an obvious indicator of the effect of Non Sea Salt (NSS) fraction of aerosols. Any particular component $(\mathrm{X})$ derived from sea salt sources gets enriched only due to the effect of the same component derived from anthropogenic sources. (Das et al., 2011).

Using the relationship shown in table 7 and table 8, the nss (\%) fraction for different ions were calculated as shown in table 9 and table 10. All ratios were higher than the sea water ratio. This indicates that the particulates matter at four areas consists of non-marine components.

\section{$\mathrm{NO}_{3}^{-} / \mathrm{nns}-\mathrm{SO}_{4}^{2-}$ ratio}

The mass ration of $\mathrm{NO}_{3}^{-} / \mathrm{nns}^{-\mathrm{SO}_{4}^{2-}}$ has been as an index of the respective importance of mobile vs. stationary sources of sulfur and nitrogen in atmosphere (Wang et al., 2017).

From table 9 in this study, all the ratios were lower than one except for TSP in Tabbin, the low mass ratios implicit stationary emissions were triumphant for Atmospheric Particulate Matter. 
Journal of Environmental Sciences (JES)

Institute of Environmental Studies and Research, Ain Shams University

Elmotily, Hossam et al.

Table(7): Evaluation of sea salt ration, NSS and EF at Autumn season

\begin{tabular}{|c|c|c|c|c|c|c|}
\hline Ratio & $\begin{array}{c}\text { Particulates } \\
\text { type }\end{array}$ & $\mathrm{Cl}^{-} / \mathrm{Na}^{+}$ & $\mathrm{SO}_{4}^{2-} / \mathrm{Na}^{+}$ & $\mathrm{Ca}^{2+} / \mathrm{Na}^{+}$ & $\mathrm{K}^{+} / \mathrm{Na}^{+}$ & $\mathrm{Mg}^{2+} / \mathrm{Na}^{+}$ \\
\hline \multirow{2}{*}{ Tabbin } & TSP & 3.71 & 7.90 & 0.33 & 5.01 & 0.27 \\
\hline & $\mathrm{PM}_{10}$ & 5.35 & 11.58 & 0.47 & 5.84 & 0.33 \\
\hline \multirow{2}{*}{ Helwan } & TSP & 18.56 & 11.55 & 2.75 & 10.80 & 1.29 \\
\hline & $\mathrm{PM}_{10}$ & 6.78 & 6.08 & 1.45 & 3.48 & 0.55 \\
\hline \multirow{2}{*}{$\begin{array}{c}\text { Shobra } \\
\text { Elkhimah }\end{array}$} & TSP & 0.99 & 2.35 & 0.10 & 0.89 & 0.13 \\
\hline & $\mathrm{PM}_{10}$ & 5.05 & 4.11 & 0.49 & 3.26 & 0.33 \\
\hline \multicolumn{2}{|c|}{$\begin{array}{c}\text { Sea ratio } \\
\text { (N. Das et al., 2011) }\end{array}$} & 1.167 & 0.125 & 0.044 & 0.227 & 0.022 \\
\hline \multicolumn{7}{|c|}{ Non sea salt (NSS) (\%) } \\
\hline & $\begin{array}{c}\text { Particulates } \\
\text { type }\end{array}$ & $\mathrm{Cl}^{-}$ & $\mathrm{SO}_{4}^{2-}$ & $\mathrm{Ca}^{2+}$ & $\boldsymbol{K}^{+}$ & $M g^{2+}$ \\
\hline \multirow{2}{*}{ Tabbin } & TSP & 16.03 & 30.05 & 0.46 & 22.16 & 1.11 \\
\hline & $\mathrm{PM}_{10}$ & 12.44 & 24.79 & 0.57 & 13.81 & 0.73 \\
\hline \multirow{2}{*}{ Helwan } & TSP & 54.39 & 30.63 & 7.45 & 31.74 & 3.73 \\
\hline & $\mathrm{PM}_{10}$ & 36.87 & 27.22 & 6.80 & 19.03 & 2.95 \\
\hline \multirow{2}{*}{$\begin{array}{l}\text { Shobra } \\
\text { Elkhimah }\end{array}$} & TSP & 19.00 & 26.06 & -2.72 & 18.61 & 2.48 \\
\hline & $\mathrm{PM}_{10}$ & 16.53 & 9.89 & 0.88 & 10.81 & 1.05 \\
\hline
\end{tabular}


Elmotily, Hossam et al.

Cont. Table(7): Evaluation of sea salt ration, NSS and EF at Autumn season

\begin{tabular}{|c|c|c|c|c|c|c|}
\hline \multicolumn{7}{|c|}{ Enrichment Factor (EF) with respect to sea water } \\
\hline \multirow{2}{*}{ Tabbin } & $\begin{array}{c}\text { Particulates } \\
\text { type }\end{array}$ & $\boldsymbol{C l}^{-}$ & $\mathbf{S O}_{4}^{2-}$ & $\boldsymbol{C a}^{2+}$ & $\boldsymbol{K}^{+}$ & $\boldsymbol{M g}^{2+}$ \\
\cline { 2 - 7 } & $\mathrm{TSP}$ & 29.72 & 63.18 & 2.64 & 40.05 & 2.17 \\
\hline \multirow{2}{*}{ Helwan } & $\mathrm{PM}_{10}$ & 42.80 & 92.65 & 3.75 & 46.74 & 2.63 \\
\cline { 2 - 7 } & $\mathrm{TSP}$ & 148.49 & 92.42 & 22.01 & 86.44 & 10.29 \\
\hline $\begin{array}{c}\text { Shobra } \\
\text { Elkhimah }\end{array}$ & $\mathrm{PM}_{10}$ & 54.23 & 48.64 & 11.63 & 27.83 & 4.43 \\
\cline { 2 - 7 } & $\mathrm{PM}_{10}$ & 7.90 & 18.80 & 0.83 & 7.11 & 1.08 \\
\hline
\end{tabular}

Table(8): Evaluation of sea salt ration, NSS and EF at Spring season

\begin{tabular}{|c|c|c|c|c|c|c|}
\hline Ratio & $\begin{array}{c}\text { Particulates } \\
\text { type }\end{array}$ & $\mathrm{Cl}^{-} / \mathrm{Na}^{+}$ & $\mathrm{SO}_{4}^{2-} / \mathrm{Na}^{+}$ & $\mathrm{Ca}^{2+} / \mathrm{Na}^{+}$ & $K^{+} / \mathrm{Na}^{+}$ & $\mathrm{Mg}^{2+} / \mathrm{Na}^{+}$ \\
\hline \multirow{2}{*}{ Tabbin } & TSP & 7.90 & 3.71 & 5.01 & 0.33 & 0.27 \\
\hline & PM10 & 11.50 & 5.34 & 5.83 & 0.46 & 0.33 \\
\hline \multirow{2}{*}{ Helwan } & TSP & 6.30 & 10.09 & 5.66 & 1.42 & 0.66 \\
\hline & PM10 & 2.35 & 0.99 & 0.89 & 0.10 & 0.13 \\
\hline \multirow{2}{*}{$\begin{array}{c}\text { Shobra } \\
\text { Elkhimah }\end{array}$} & TSP & 2.35 & 0.99 & 0.89 & 0.10 & 0.13 \\
\hline & PM10 & 4.11 & 5.05 & 3.26 & 0.49 & 0.33 \\
\hline \multicolumn{2}{|c|}{$\begin{array}{c}\text { Sea ratio } \\
\text { (N. Das et al., 2011) }\end{array}$} & 1.167 & 0.125 & 0.044 & 0.227 & 0.022 \\
\hline \multicolumn{7}{|c|}{ Non sea salt (nss) (\%) } \\
\hline & $\begin{array}{c}\text { Particulates } \\
\text { type }\end{array}$ & $\mathrm{Cl}^{-}$ & $\mathrm{SO}_{4}^{2-}$ & $C a^{2+}$ & $\boldsymbol{K}^{+}$ & $M g^{2+}$ \\
\hline \multirow{2}{*}{ Tabbin } & TSP & 58.47 & 31.18 & 43.11 & 0.89 & 2.17 \\
\hline & PM10 & 46.39 & 23.41 & 25.99 & 1.06 & 1.37 \\
\hline \multirow{2}{*}{ Helwan } & TSP & 21.75 & 42.22 & 23.78 & 5.07 & 2.71 \\
\hline & PM10 & 31.54 & 23.00 & 22.53 & -3.29 & 3.01 \\
\hline \multirow{2}{*}{$\begin{array}{c}\text { Shobra } \\
\text { Elkhimah }\end{array}$} & TSP & 31.54 & 23.00 & 22.53 & -3.29 & 3.01 \\
\hline & PM10 & 11.97 & 20.00 & 13.08 & 1.07 & 1.27 \\
\hline \multicolumn{7}{|c|}{ Enrichment Factor (EF) with respect to sea water } \\
\hline & $\begin{array}{c}\text { Particulates } \\
\text { type }\end{array}$ & $\mathrm{Cl}^{-}$ & $\mathrm{SO}_{4}^{2-}$ & $C a^{2+}$ & $\boldsymbol{K}^{+}$ & $M g^{2+}$ \\
\hline \multirow{2}{*}{ Tabbin } & TSP & 63.18 & 29.72 & 40.05 & 2.64 & 2.17 \\
\hline & PM10 & 91.97 & 42.70 & 46.65 & 3.71 & 2.61 \\
\hline \multirow{2}{*}{ Helwan } & TSP & 50.42 & 80.75 & 45.27 & 11.39 & 5.30 \\
\hline & PM10 & 18.80 & 7.90 & 7.11 & 0.83 & 1.08 \\
\hline \multirow{2}{*}{$\begin{array}{c}\text { Shobra } \\
\text { Elkhimah } \\
\end{array}$} & TSP & 18.80 & 7.90 & 7.11 & 0.83 & 1.08 \\
\hline & PM10 & 32.90 & 40.38 & 26.10 & 3.91 & 2.67 \\
\hline
\end{tabular}

Vol.(50); Iss.(3); No.(1); Mar..2021 
In the current study, the $\mathrm{NO}_{3}^{-} / \mathrm{SO}_{4}^{2-}$ ratios in Tabbin (0.94-1.19) were higher than in Helwan (0.42-0.95) and Shobra Elkhimah (0.42-0.58) for two types of particles in both spring and autumn, indication that the contribution of stationary emission to total particles in Tabbin were higher than in Helwan and Shobra Elkhimah.

Table(9): Mass ration of $\mathrm{NO}_{3}^{-} / \mathrm{NSS}_{-} \mathrm{SO}_{4}^{2-}$

\begin{tabular}{|c|c|c|c|}
\hline \multirow{2}{*}{ area } & \multirow{2}{*}{ Particulates type } & \multicolumn{2}{|c|}{$\mathrm{NO}_{3}^{-} / \mathrm{SO}_{4}^{2-}$} \\
\hline & & Autumn & Spring \\
\hline \multirow{2}{*}{ Tabbin } & TSP & 1.19 & 1.19 \\
\hline & PM10 & 0.94 & 0.95 \\
\hline \multirow{2}{*}{ Helwan } & TSP & 0.42 & 0.53 \\
\hline & PM10 & 0.95 & 0.42 \\
\hline \multirow{2}{*}{$\begin{array}{l}\text { Shobra } \\
\text { Elkhimah }\end{array}$} & TSP & 0.42 & 0.42 \\
\hline & PM10 & 0.58 & 0.58 \\
\hline $\mathrm{PM}_{10}$ : & $\begin{array}{r}\text { TSP: Total } \\
\text { late Matter with a } \\
\mathrm{NSS}\end{array}$ & $\begin{array}{l}\text { ed Particul } \\
\text { ynamic diat } \\
\text { ea Salt }\end{array}$ & $\mathrm{n} 10 \mu \mathrm{m}$ \\
\hline
\end{tabular}

$\circ$ Correlation Coefficient: Table 10 represent bivariate Pearson correlations between soluble ions in particulate matter from different areas in Cairo at autumn and spring seasons. 
Journal of Environmental Sciences (JES)

Institute of Environmental Studies and Research, Ain Shams University

Elmotily, Hossam et al.

Table(10): Bivariate Pearson correlations between heavy metals in TSP and PM10

\begin{tabular}{|c|c|c|c|c|c|c|c|c|c|}
\hline \multicolumn{10}{|c|}{ Tabbin } \\
\hline & $\mathrm{SO}_{4}^{-}$ & $\mathrm{NO}_{3}^{-}$ & $F^{-}$ & $\mathrm{Cl}^{-}$ & $\mathrm{NH}_{4}^{+}$ & $\mathrm{Na}^{+}$ & $K^{+}$ & $\mathrm{Ca}^{2+}$ & $M_{g}{ }^{2+}$ \\
\hline \multicolumn{10}{|c|}{ TSP } \\
\hline $\mathrm{SO}_{4}^{2-}$ & 1 & & & & & & & & \\
\hline $\mathrm{NO}_{3}^{-}$ & $.843^{*}$ & 1 & & & & & & & \\
\hline$F^{-}$ & .690 & $.911^{*}$ & 1 & & & & & & \\
\hline $\mathrm{Cl}^{-}$ & .665 & .266 & -.072 & 1 & & & & & \\
\hline $\mathrm{NH}_{4}^{+}$ & $.820^{*}$ & .735 & $.835^{*}$ & .213 & 1 & & & & \\
\hline $\mathrm{Na}^{+}$ & .325 & .499 & .152 & .419 & -.180 & 1 & & & \\
\hline$K^{+}$ & $.933^{* * *}$ & $848^{*}$ & $.845^{*}$ & .386 & $.968^{* * *}$ & .062 & 1 & & \\
\hline $\mathrm{Ca}^{2+}$ & .712 & .232 & .134 & .769 & .617 & -.187 & .640 & 1 & \\
\hline$M g^{2+}$ & $.824^{*}$ & .687 & .341 & $.844^{*}$ & .354 & .742 & .575 & .509 & 1 \\
\hline \multicolumn{10}{|c|}{ PM10 $_{10}$} \\
\hline $\mathrm{SO}_{4}^{2-}$ & 1 & & & & & & & & \\
\hline $\mathrm{NO}_{3}^{-}$ & .471 & 1 & & & & & & & \\
\hline$F^{-}$ & $.991^{* * *}$ & .421 & 1 & & & & & & \\
\hline $\mathrm{Cl}^{-}$ & .796 & -.134 & $.842^{*}$ & 1 & & & & & \\
\hline $\mathrm{NH}_{4}^{+}$ & .633 & .794 & .666 & .279 & 1 & & & & \\
\hline $\mathrm{Na}^{+}$ & .541 & $.859^{*}$ & .439 & -.055 & .467 & 1 & & & \\
\hline$K^{+}$ & $.855^{*}$ & .283 & .790 & .665 & .198 & .615 & 1 & & \\
\hline $\mathrm{Ca}^{2+}$ & $.986^{* * *}$ & .380 & $.999^{* *}$ & $.865^{*}$ & .645 & .400 & .785 & 1 & \\
\hline$M g^{2+}$ & $.923^{* *}$ & .101 & $943^{* * *}$ & $965^{* *}$ & .410 & .207 & .805 & $.956^{* * *}$ & 1 \\
\hline
\end{tabular}

Vol.(50); Iss.(3); No.(1); Mar..2021 
Journal of Environmental Sciences (JES)

Institute of Environmental Studies and Research, Ain Shams University

Elmotily, Hossam et al.

Cont. Table(10): Bivariate Pearson correlations between heavy metals in TSP and PM10

\begin{tabular}{|c|c|c|c|c|c|c|c|c|}
\hline \multicolumn{9}{|c|}{ Helwan } \\
\hline & $\mathrm{SO}_{4}^{2-}$ & $\mathrm{NO}_{3}^{-}$ & $F^{-}$ & $\mathrm{Cl}^{-}$ & $\mathrm{NH}_{4}^{+}$ & $K^{+}$ & $\mathrm{Ca}^{2+}$ & $M g^{2+}$ \\
\hline \multicolumn{9}{|c|}{ TSP } \\
\hline $\mathrm{SO}_{4}^{2-}$ & 1 & & & & & & & \\
\hline $\mathrm{NO}_{3}^{-}$ & $.873^{*}$ & 1 & & & & & & \\
\hline$F^{-}$ & $.998^{* * *}$ & $.899^{*}$ & 1 & & & & & \\
\hline $\mathrm{Cl}^{-}$ & $1.000^{* *}$ & $.875^{*}$ & $.999^{* * *}$ & 1 & & & & \\
\hline $\mathrm{NH}_{4}^{+}$ & .045 & .523 & .102 & .049 & 1 & & & \\
\hline$K^{+}$ & $.998^{* *}$ & $.844^{*}$ & $.994^{* *}$ & $.998^{* * *}$ & -.011 & 1 & & \\
\hline $\mathrm{Ca}^{2+}$ & $.999^{* * *}$ & $.851^{*}$ & $.995^{* * *}$ & $.999^{* * *}$ & .002 & $1.000^{* *}$ & 1 & \\
\hline$M g^{2+}$ & $.998^{* *}$ & $.842^{*}$ & $.993^{* *}$ & $.998^{* * *}$ & -.015 & $1.000^{* * *}$ & $1.000^{* *}$ & 1 \\
\hline \multicolumn{9}{|c|}{$\mathrm{PM}_{10}$} \\
\hline $\mathrm{SO}_{4}^{2-}$ & 1 & & & & & & & \\
\hline $\mathrm{NO}_{3}^{-}$ & $.999^{* * *}$ & 1 & & & & & & \\
\hline$F^{-}$ & $.999^{* * *}$ & $.997^{* *}$ & 1 & & & & & \\
\hline $\mathrm{Cl}^{-}$ & $.999^{* *}$ & $.997^{* * *}$ & $1.000^{* * *}$ & 1 & & & & \\
\hline $\mathrm{NH}_{4}^{+}$ & $1.000^{* *}$ & $1.000^{* *}$ & $.997^{* * *}$ & $.998^{* * *}$ & 1 & & & \\
\hline$K^{+}$ & $1.000^{\text {*** }}$ & $.998^{* *}$ & $1.000^{* * *}$ & $1.000^{* * *}$ & $.998^{* * *}$ & 1 & & \\
\hline $\mathrm{Ca}^{2+}$ & $1.000^{* * *}$ & $.999^{* * *}$ & $.999^{* * *}$ & $.999^{* * *}$ & $1.000^{* *}$ & $1.000^{* * *}$ & 1 & \\
\hline$M g^{2+}$ & $1.000^{* * *}$ & $1.000^{* *}$ & $.999^{* * *}$ & $.999^{* * *}$ & $1.000^{* *}$ & $.999^{* *}$ & $1.000^{* *}$ & 1 \\
\hline
\end{tabular}


Journal of Environmental Sciences (JES)

Institute of Environmental Studies and Research, Ain Shams University

Elmotily, Hossam et al.

Cont. Table(10): Bivariate Pearson correlations between heavy metals in TSP and PM10

\begin{tabular}{|c|c|c|c|c|c|c|c|c|}
\hline \multicolumn{9}{|c|}{ Shobra Elkhimah } \\
\hline & $\mathrm{SO}_{4}^{2-}$ & $\mathrm{NO}_{3}^{-}$ & $F^{-}$ & $\mathrm{Cl}^{-}$ & $\mathrm{NH}_{4}^{+}$ & $K^{+}$ & $\mathrm{Ca}^{2+}$ & $\mathrm{Mg}^{2+}$ \\
\hline \multicolumn{9}{|c|}{ TSP } \\
\hline $\mathrm{SO}_{4}^{2-}$ & 1 & & & & & & & \\
\hline $\mathrm{NO}_{3}^{-}$ & -.189 & 1 & & & & & & \\
\hline$F^{-}$ & -.375 & $.981^{* * *}$ & 1 & & & & & \\
\hline $\mathrm{Cl}^{-}$ & .700 & $-.834^{*}$ & $-.924^{* *}$ & 1 & & & & \\
\hline $\mathrm{NH}_{4}^{+}$ & .686 & $-.844^{*}$ & $-.932^{* *}$ & $1.000^{* *}$ & 1 & & & \\
\hline$K^{+}$ & $.980^{* *}$ & -.383 & -.553 & $.829^{*}$ & $.818^{*}$ & 1 & & \\
\hline $\mathrm{Ca}^{2+}$ & .780 & -.762 & $-.873^{*}$ & $.993^{* *}$ & $.990^{* *}$ & $.890^{*}$ & 1 & \\
\hline $\mathrm{Mg}^{2+}$ & .738 & -.802 & $-.902^{*}$ & $.999^{* * *}$ & $.997^{* *}$ & $.858^{*}$ & $.998^{* *}$ & 1 \\
\hline \multicolumn{9}{|c|}{$\mathbf{P M}_{10}$} \\
\hline $\mathrm{SO}_{4}^{2-}$ & 1 & & & & & & & \\
\hline $\mathrm{NO}_{3}^{-}$ & .786 & 1 & & & & & & \\
\hline$F^{-}$ & -.535 & .101 & 1 & & & & & \\
\hline $\mathrm{Cl}^{-}$ & $.979^{* * *}$ & $.895^{*}$ & -.353 & 1 & & & & \\
\hline $\mathrm{NH}_{4}^{+}$ & $.998^{* * *}$ & $.821^{*}$ & -.485 & $.989^{* * *}$ & 1 & & & \\
\hline$K^{+}$ & $.890^{*}$ & $.982^{* * *}$ & -.091 & $.964^{* *}$ & $.915^{*}$ & 1 & & \\
\hline $\mathrm{Ca}^{2+}$ & $.999^{* *}$ & $.814^{*}$ & -.496 & $.988^{* *}$ & $1.000^{* *}$ & $.910^{*}$ & 1 & \\
\hline $\mathrm{Mg}^{2+}$ & .359 & $.859^{*}$ & .597 & .540 & .412 & .745 & .401 & 1 \\
\hline
\end{tabular}


Table(10): Bivariate Pearson correlations between heavy metals in TSP and PM10

\begin{tabular}{|c|c|c|c|c|c|c|c|}
\hline \multicolumn{8}{|c|}{ Fifth settlement } \\
\hline & $\mathrm{SO}_{4}^{2-}$ & $\mathrm{NO}_{3}^{-}$ & $F^{-}$ & $\mathrm{Cl}^{-}$ & $K^{+}$ & $\mathrm{Ca}^{2+}$ & $\mathrm{Mg}^{2+}$ \\
\hline \multicolumn{8}{|c|}{ TSP } \\
\hline $\mathrm{SO}_{4}^{2-}$ & 1 & & & & & & \\
\hline $\mathrm{NO}_{3}^{-}$ & $.979^{* * *}$ & 1 & & & & & \\
\hline$F^{-}$ & $.971^{* *}$ & $.999^{* * *}$ & 1 & & & & \\
\hline $\mathrm{Cl}^{-}$ & -.418 & -.593 & -.622 & 1 & & & \\
\hline$K^{+}$ & $.994^{* *}$ & $.996^{* *}$ & $.992^{* *}$ & -.518 & 1 & & \\
\hline $\mathrm{Ca}^{2+}$ & -.781 & $-.891^{*}$ & $-.907^{*}$ & $.894^{*}$ & $-.846^{*}$ & 1 & \\
\hline$M g^{2+}$ & $981^{* *}$ & $1.000^{* *}$ & $.999^{* *}$ & -.586 & $.997^{* * *}$ & $-.887^{*}$ & 1 \\
\hline \multicolumn{8}{|c|}{$\mathbf{P M}_{10}$} \\
\hline $\mathrm{SO}_{4}^{2-}$ & 1 & & & & & & \\
\hline $\mathrm{NO}_{3}^{-}$ & $.979^{* * *}$ & 1 & & & & & \\
\hline$F^{-}$ & $973^{* *}$ & $1.000^{* *}$ & 1 & & & & \\
\hline $\mathrm{Cl}^{-}$ & .758 & .608 & .588 & 1 & & & \\
\hline$K^{+}$ & $.999^{* *}$ & $.988^{* *}$ & $.984^{* *}$ & .723 & 1 & & \\
\hline $\mathrm{Ca}^{2+}$ & .373 & .174 & .149 & $.887^{*}$ & .323 & 1 & \\
\hline$M g^{2+}$ & $968^{* * *}$ & $.999^{* *}$ & $1.000^{* * *}$ & .571 & $.980^{* * *}$ & .129 & 1 \\
\hline
\end{tabular}

*. Correlation is significant at the 0.05 level (2-tailed).

**. Correlation is significant at the 0.01 level (2-tailed).

\section{CONCLUSION}

- In spring, the maximum concentration in the TSP of $\mathrm{Co}, \mathrm{Cu}, \mathrm{Fe}, \mathrm{Mg}$ and $\mathrm{Ni}$ are recorded in Shobra Elkhimah, $\mathrm{Al}, \mathrm{Mn}$ and $\mathrm{Zn}$ are found in Helwan, whereas in autumn, the maximum concentration in the TSP of $\mathrm{Co}, \mathrm{Cu}, \mathrm{Fe}$ and $\mathrm{Ni}$ is recorded in Shobra Elkhimah, $\mathrm{Al}, \mathrm{Cu}, \mathrm{Mn}$ and $\mathrm{Zn}$ are found in Helwan. At both seasons, $\mathrm{Pb}$ is found in Tabbin and $\mathrm{Cr}$ is not found in TSP 
for all filters sample. On the other hand, in spring, the maximum concentration of $\mathrm{NO}_{3}^{-}, \mathrm{Cl}^{-}$, and $\mathrm{Ca}^{2+}$ are found in Tabbin, $\mathrm{F}^{-}, \mathrm{Na}^{+}$, and $\mathrm{Mg}^{2+}$ are found in Shobra Elkhimah and $\mathrm{SO}_{4}^{-}$and $\mathrm{K}^{+}$are recorded in Helwan, while in autumn, $\mathrm{SO}_{4}^{-}, \mathrm{NO}_{3}^{-}, \mathrm{F}^{-}, \mathrm{K}^{+}, \mathrm{Ca}^{2+}$ and $\mathrm{Mg}^{2+}$ are found in Helwan, $\mathrm{Cl}^{-}$and $\mathrm{Na}^{+}$are recorded in Shobra Elkhimah. $\mathrm{NH}_{4}^{+}$is found only in Tabbin. $\mathrm{Na}^{+}$is not recorded in Fifth Settlement.

- The maximum concentration in the $\mathrm{PM}_{10}$ of $\mathrm{Al}, \mathrm{Co}, \mathrm{Cu}, \mathrm{Fe}$ and $\mathrm{Mg}$ are found in Shobra Elkhimah, $\mathrm{Ni}$ and $\mathrm{Pb}$ are found in Tabbin, Mn is found only in Fifth Settlement and $\mathrm{Zn}$ is found only in Helwan. While in autumn, the maximum concentration in the $\mathrm{PM}_{10}$ of $\mathrm{Al}, \mathrm{Co}, \mathrm{Fe}, \mathrm{Mn}, \mathrm{Ni}$ and $\mathrm{Zn}$ is recorded in Helwan, $\mathrm{Pb}$ is found only in Tabbin, $\mathrm{Cu}$ is found in Shobra Elkhimah and $\mathrm{Mg}$ is found in Fifth Settlement. At both seasons, and $\mathrm{Cr}$ is not found in $\mathrm{PM}_{10}$ for all filters sample. On the other hand, in spring, the maximum concentration of $\mathrm{SO}_{4}^{-}, \mathrm{NO}_{3}^{-}, \mathrm{Cl}^{-}, \mathrm{Na}^{+}, \mathrm{Ca}^{2+}$ and $\mathrm{Mg}^{2+}$ are found in Tabbin, $\mathrm{F}^{-}$is found in Shobra Elkhimah and $\mathrm{K}^{+}$is recorded in Helwan. $\mathrm{NH}_{4}^{+}$is found only in Tabbin also. $\mathrm{Na}^{+}$is not recorded in Fifth 
Settlement also, while in autumn, $\mathrm{SO}_{4}^{-}, \mathrm{NO}_{3}^{-}, \mathrm{F}^{-}, \mathrm{Cl}^{-}, \mathrm{Na}^{+}, \mathrm{K}^{+}, \mathrm{Ca}^{2+}$ and $\mathrm{Mg}^{2+}$ are found in Helwan,. $\mathrm{NH}_{4}^{+}$is found only in Tabbin also. $\mathrm{Na}^{+}$ is not recorded in Fifth Settlement also.

- Generally, it may be concluded that all heavy metals increase in Tabbin, Helwan, Shobra Elkhimah and Fifth Settlement in spring, otherwise decrease in autumn. On the contrary, all water-soluble ions increase in autumn in study areas and decrease in spring.

- The calculated EF revealed that all analyzed elements and Soluble ions are of anthropogenic origin

- From the point of occupational health, all areas are ranging from moderate to danger for both spring and autumn.

\section{RECOMMENDATION}

- From point of mitigation, other roads should be structured to motivate the air motion

- The landscape should be taken into consideration as an additional lung to the present citizens because of low pollution.

- Madinaty or El-Rehab areas should be taken as a reference areas instead of the Fifth Settlement.

- The results obtained from this project can be the base foundation of a new project funded by the Egyptian government to conduct the same 
measurements for heavy metals and water-soluble ions in several area all over Egypt a data base for the concentration of Such pollutants in several areas in Egypt can be studied.

- The ministry of environment, ministry of health and ministry commerce can cooperate in a joint study to estimate the impact of such pollutants on the human health, local environment and Egyptian economy

- Perform continuous monitoring programs for the ambient air in areas under study.

- Applying stringent legislations through connecting the stack source emissions for different industrial processes to national network for air quality monitoring using CEMS (continuous emission monitoring systems).

- Applying treatment or filter systems for different industrial complexes such as electro-precipitators, bag filters and others.

- Finding other routes in order to decrease vehicles emission load resulted from heavy traffic roads.

- Applying precancerous monitoring programs in residential area, as we all know that early detection of problems is very best way to treat before developing.

- Further researches are required to draw a clearer picture for the current situation in terms of air quality and health impact of hazardous air pollutants. 
- Air quality dispersion models have the ability to predict the future air quality conditions using a mathematical tool through using he collected data as an input data for the model which can predict the future conditions for the study area

- The study areas are highly populated area and also with high density, this study recommends to either reduce the number of industrial facilities in the area or to prevent any new industrial facilities installation.

Encourage the industrializing and using the electric traffics for people with tax free benefits or obligation for school buses for example

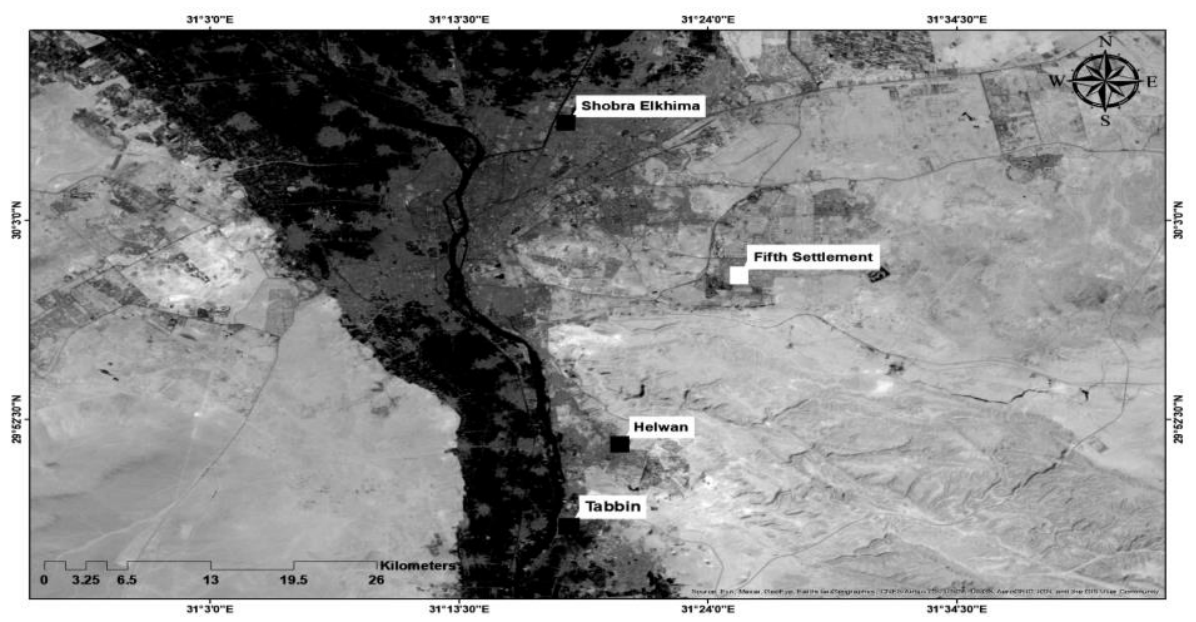

Figure(1): Map of the study area where the black spots indicate industrial sites and the white spot indicate reference area. 
Journal of Environmental Sciences (JES)

Institute of Environmental Studies and Research, Ain Shams University

Elmotily, Hossam et al.
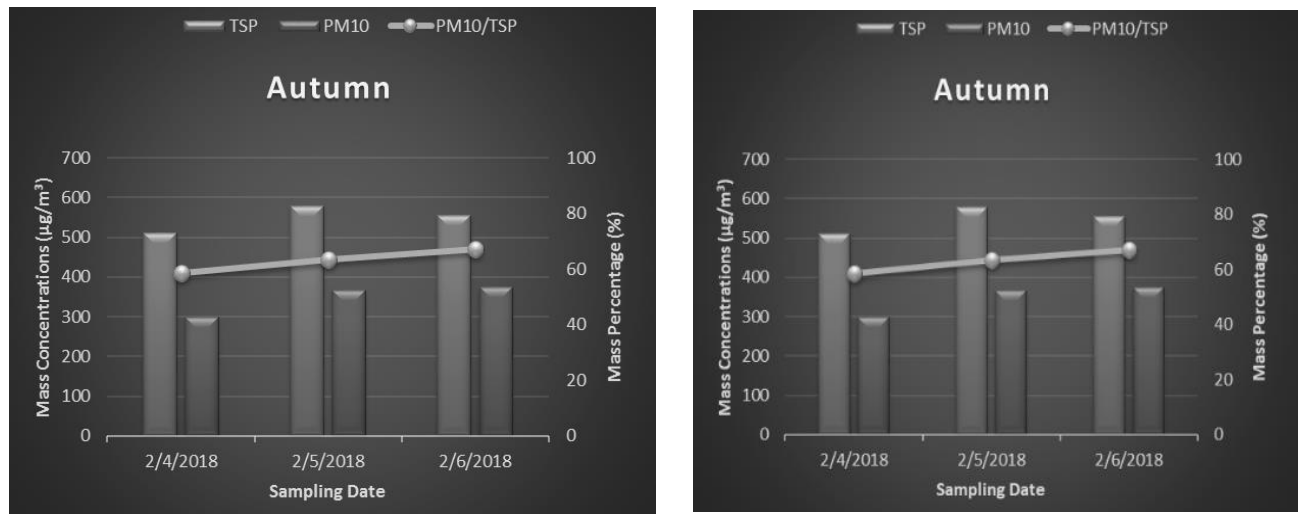

TSP: Total suspended Particulate

PM10: particulate matter with an aerodynamic diameter less than $10 \mu \mathrm{m}$

Figure(2): Time series of TSP and PM10 mass concentration and the mass percentages for PM10/TSP in different seasons for Tabbin

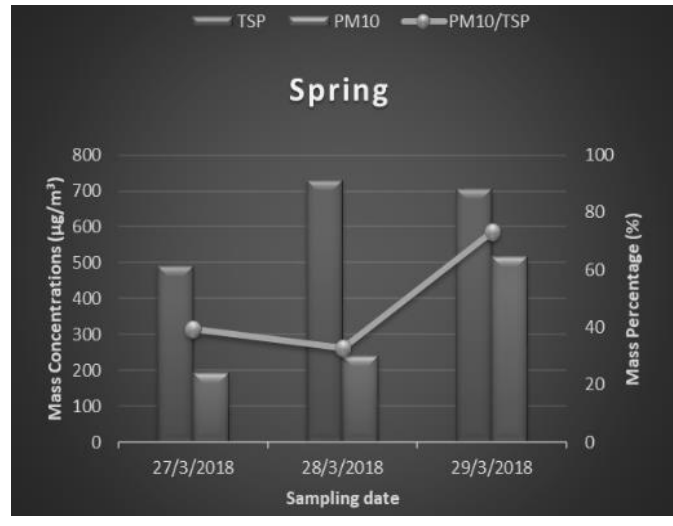

TSP: Total suspended Particulate

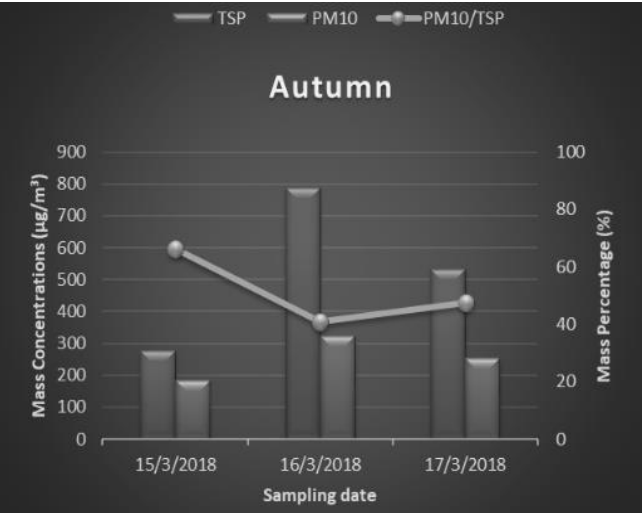

PM10: particulate matter with an aerodynamic diameter less than $10 \mu \mathrm{m}$ 
Figure(3): Time series of TSP and PM10 mass concentration and the mass percentages for PM10/TSP in different seasons for Helwan

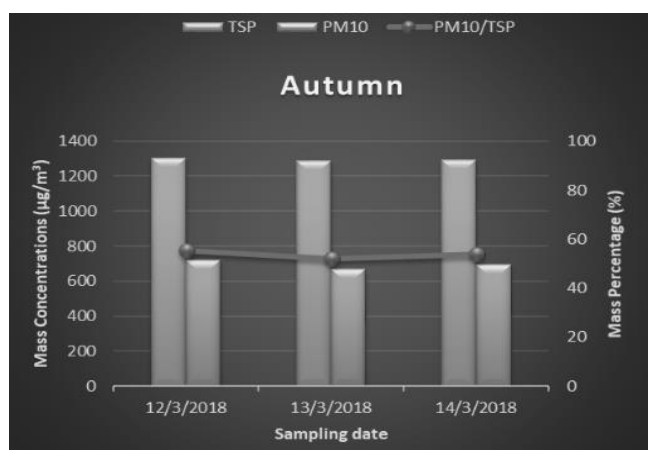

TSP: Total suspended Particulate

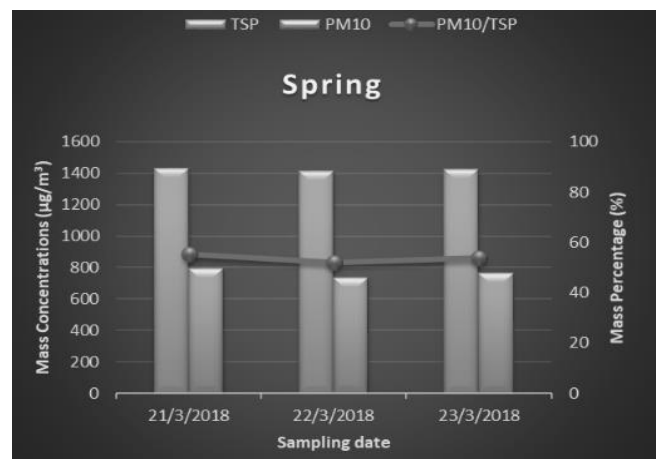

PM10: particulate matter with an aerodynamic diameter less than $10 \mu \mathrm{m}$

Figure(4): Time series of TSP and PM10 mass concentration and the mass percentages for PM10/TSP in different seasons for Shobra Elkhimah

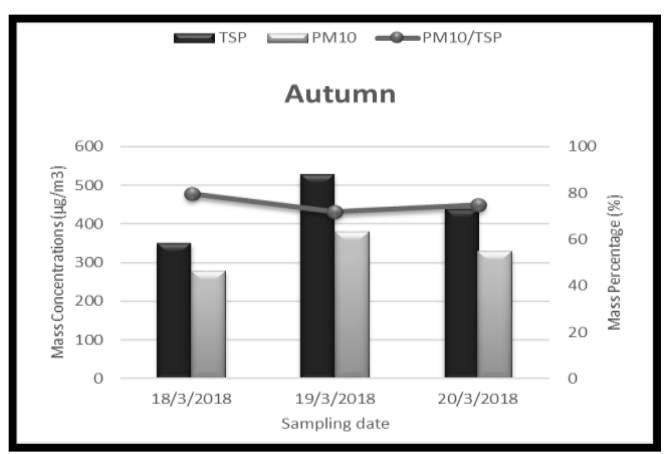

TSP: Total suspended Particulate

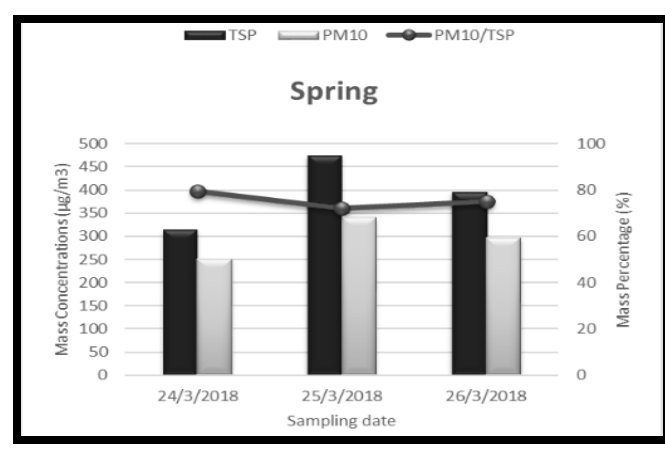

PM10: particulate matter with an aerodynamic diameter less than $10 \mu \mathrm{m}$ 
Journal of Environmental Sciences (JES)

Institute of Environmental Studies and Research, Ain Shams University

Elmotily, Hossam et al.

Figure (5): Time series of TSP and PM10 mass concentration and the mass percentages for PM10/TSP in different seasons for Fifth settlement
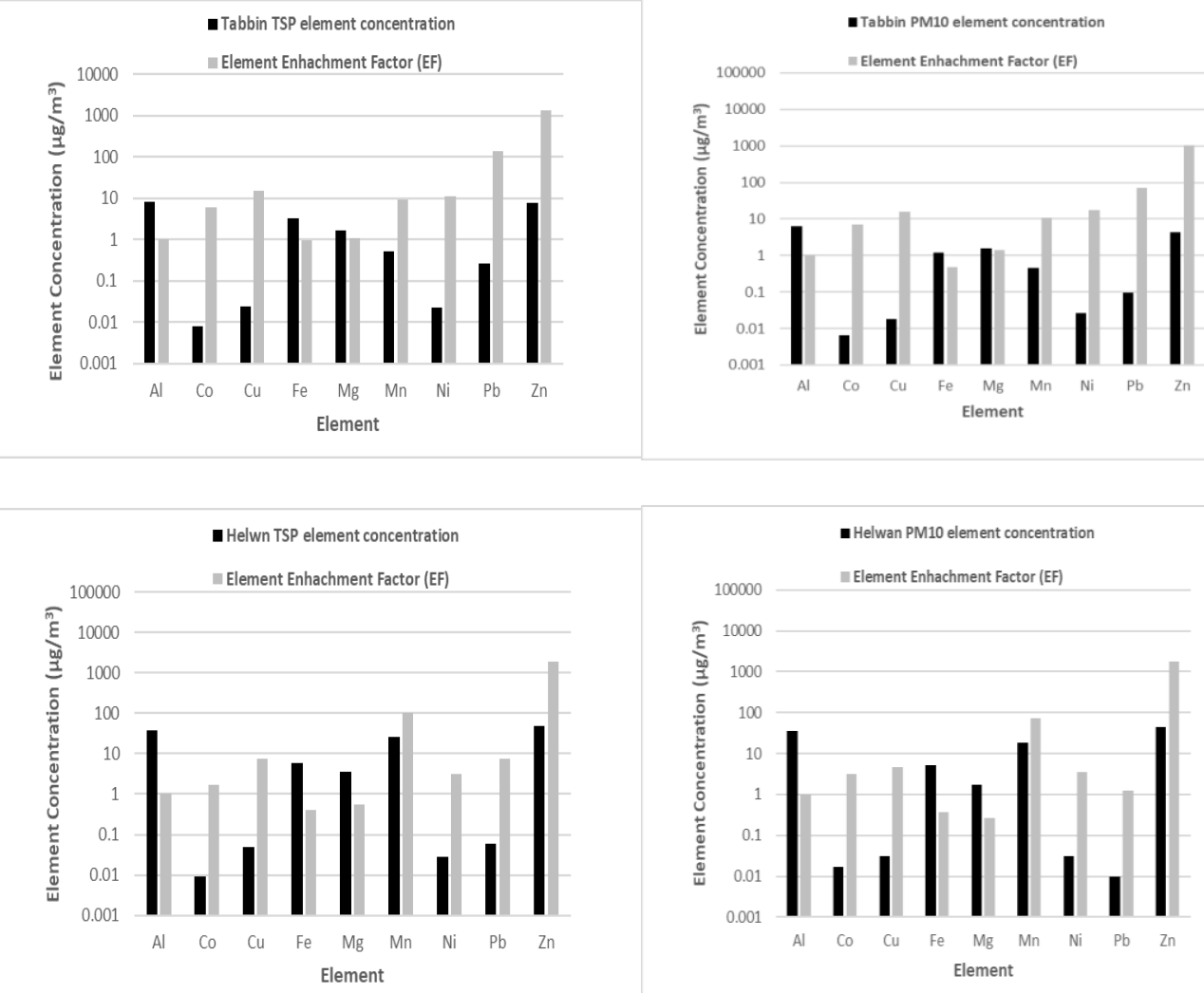

Figure(6): Heavy metals concentrations and their enrichment factors for all areas at Autumn season

Vol.(50); Iss.(3); No.(1); Mar..2021 
Journal of Environmental Sciences (JES)

Institute of Environmental Studies and Research, Ain Shams University

Elmotily, Hossam et al.
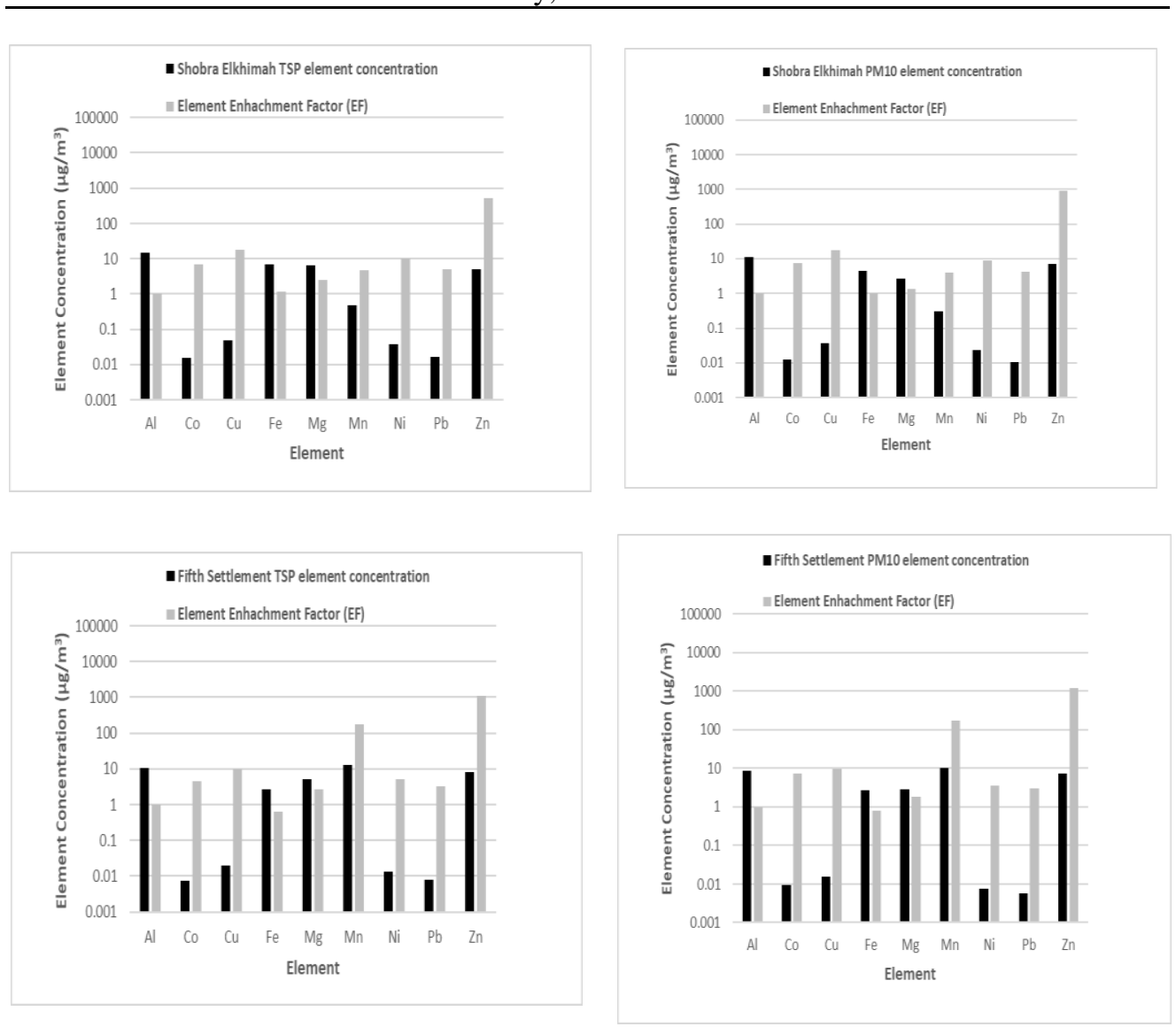

Figure(6): (Cont.) Heavy metals concentrations and their enrichment factors for all areas at Autumn season 
Journal of Environmental Sciences (JES)

Institute of Environmental Studies and Research, Ain Shams University

Elmotily, Hossam et al.
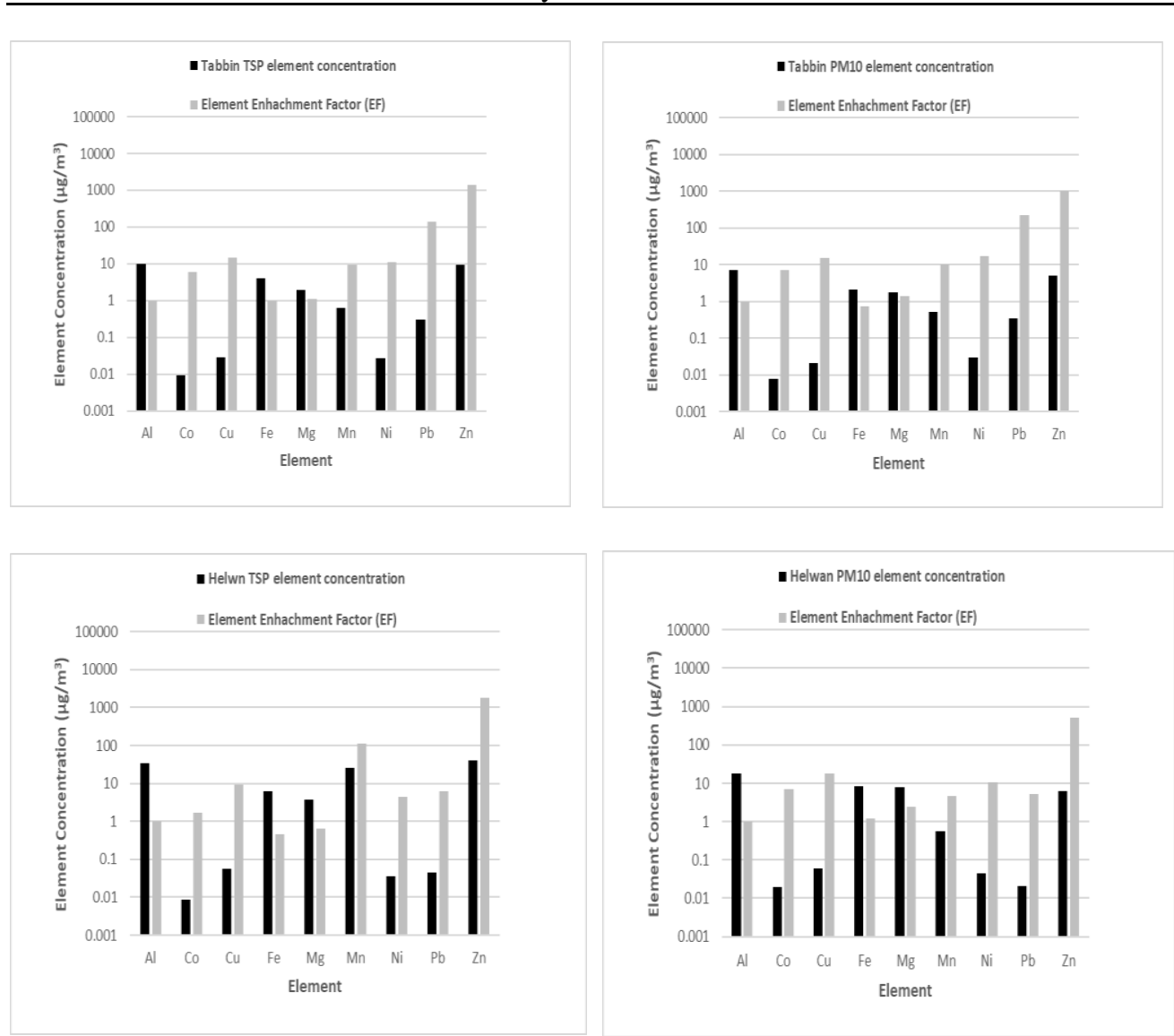

Figure(7): Heavy metals concentrations and their enrichment factors for all areas at Spring season 
Journal of Environmental Sciences (JES)

Institute of Environmental Studies and Research, Ain Shams University

Elmotily, Hossam et al.

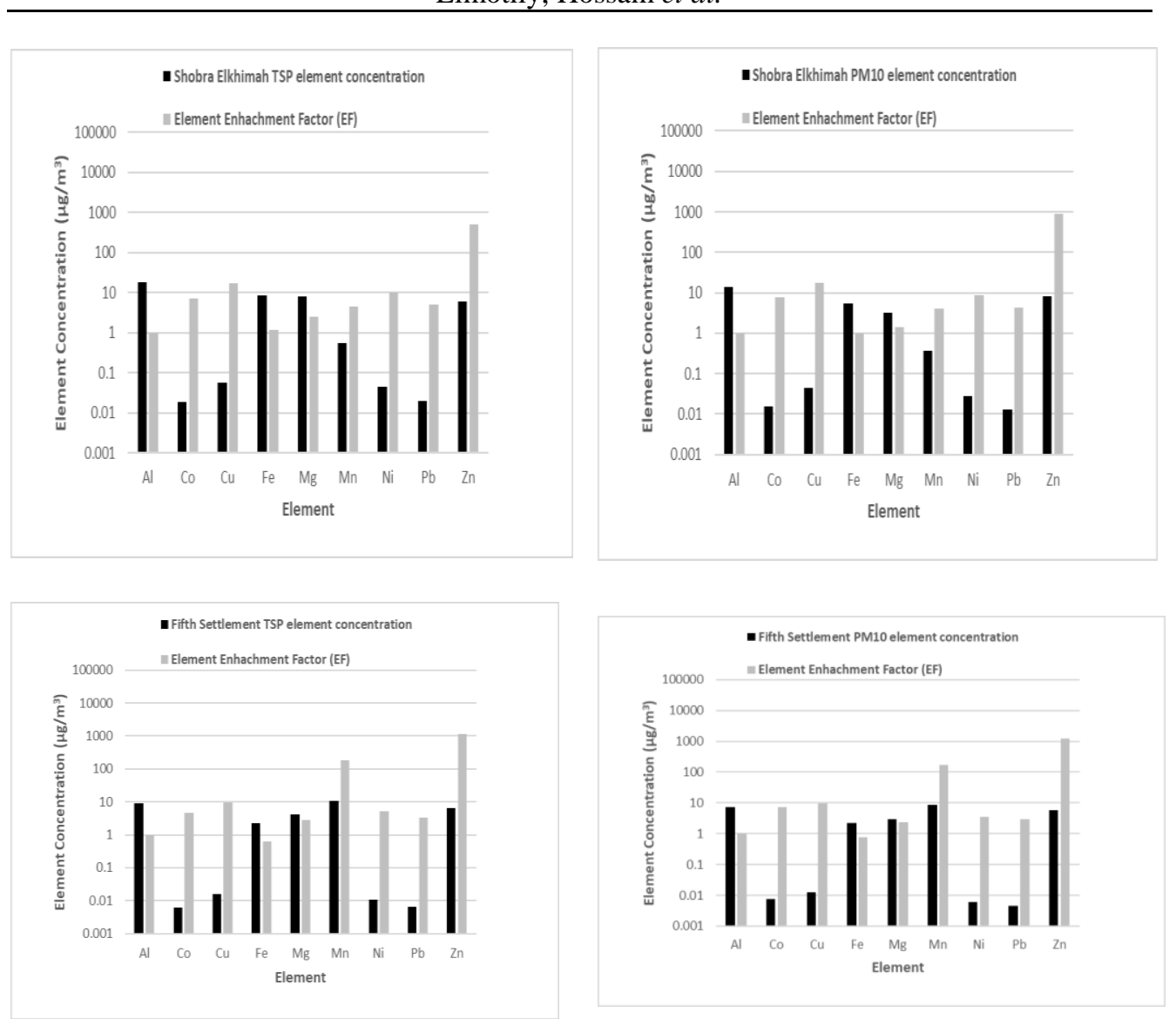

Figure (7): (Cont.) Heavy metals concentrations and their enrichment factors for all areas at Spring season 


\section{REFERENCES}

Ali-Mohamed, A.Y., (1991): Estimation of inorganic particulate matter in the atmosphere of Isa Town, Bahrain, by dry deposition. Atmospheric Environment. Part B. Urban Atmosphere, 25(3): 397-405

Andreae, M.O., Schmid, O., Yang, H., Chand, D., Yu, J.Z., Zeng, L.M. and Zhang, Y.H., (2008): Optical properties and chemical composition of the atmospheric aerosol in urban Guangzhou, China. Atmospheric Environment, 42(25): 6335-6350.

Borai E.H., Soliman A.A..,: Monitoring and statistical evaluation of heavy metals in airborne particulates in Cairo, Egypt. Journal of Chromatography A, 920, 261-269, (2001).

Das, N., Das, R., Das, S.N., Swamy, Y.V., Chaudhury, G.R. and Baral, S.S., (2011): Comparative studies of chemical composition of particulate matter between sea and remote location of eastern part of India. Atmospheric Research, 99(2): 337-343.

Geiger, A. and Cooper, J., (2010): Overview of airborne metals regulations, exposure limits, health effects, and contemporary research. Environmental Protection Agency, Air Quality: Washington, DC, USA.

Khoder, M.I., (2002): Atmospheric conversion of sulfur dioxide to particulate sulfate and nitrogen dioxide to particulate nitrate and gaseous nitric acid in an urban area. Chemosphere 49(6): 675- 684.

Kim, K.H., Kabir, E. and Kabir, S., (2015): A review on the human health impact of airborne particulate matter. Environment international, 74:136-143.

Kumar, A., (2008): Characterisation of soluble components and PAH in PM10 atmospheric particulate matter in Brisbane (Doctoral dissertation, Queensland University of Technology). 
Ma, Y., Wang, Z., Tan, Y., Xu, S., Kong, S., Wu, G., Wu, X. and Li, H., (2017): Comparison of inorganic chemical compositions of atmospheric TSP, PM10 and PM2. 5 in northern and southern Chinese coastal cities. Journal of Environmental Sciences, 55: 339-353.

Peixoto, M.S., de Oliveira Galvão, M.F. and de Medeiros, S.R.B., (2017): Cell death pathways of particulate matter toxicity. Chemosphere, 188: 32-48.

Pope, C.A., (2000): Epidemiology of fine particulate air pollution and human health: biologic mechanisms and who's at risk?. Environmental health perspectives, 108(suppl 4):713-723.

Sampler, H.V.H., 1999. Sampling of Ambient Air for Total Suspended Particulate Matter (SPM) and PM10 using High Volume (HV) Sampler.

Seinfeld, J.H., (1986): ES\&T books: atmospheric chemistry and physics of air pollution. Environmental science \& technology, 20(9):863-863.

Silva, K.K., Duarte, F.T., Jéssica Matias, T.N., Dias, S.A., Duarte, C.G., Soares, C.D., Hoelzemann, J.J., and Galvão, F.D. (2019): Physico-chemical properties and genotoxic effects of air particulate matter collected from a complex of ceramic industries. Atmospheric Pollution Research (10): 597-607

Wang, Y., Zhuang, G., Sun, Y. and An, Z., (2006): The variation of characteristics and formation mechanisms of aerosols in dust, haze, and clear days in Beijing. Atmospheric Environment, 40(34): 6579-6591.

Wardencki, W. and Bielawska, M., (2016): Particulate Material Analysis in Air. Comprehensive Analytical Chemistry, 343-367

Zhang, X.Y., Wang, Y.Q., Niu, T., Zhang, X.C., Gong, S.L., Zhang, Y.M. and Sun, J.Y., (2012): Atmospheric aerosol compositions in China: spatial/temporal variability, chemical signature, regional 
haze distribution and comparisons with global aerosols. Atmospheric Chemistry and Physics, 12(2):779-799.

Zheng, S., Pozzer, A., Cao, C.X. and Lelieveld, J., (2015): Long-term (20012012) concentrations of fine particulate matter (PM 2.5) and the impact on human health in Beijing, China. Atmospheric Chemistry and Physics, 15(10): 5715-5725.

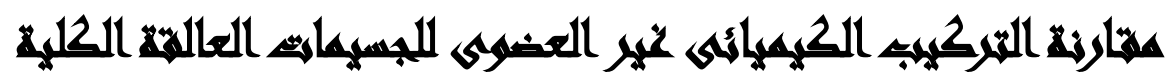

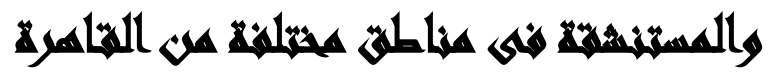

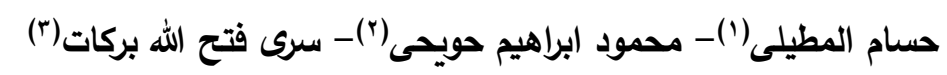

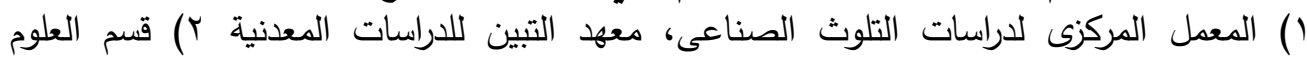

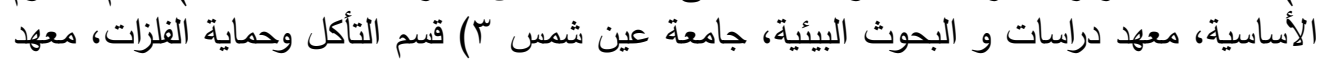

$$
\text { التبين للدراسات المعدنية }
$$

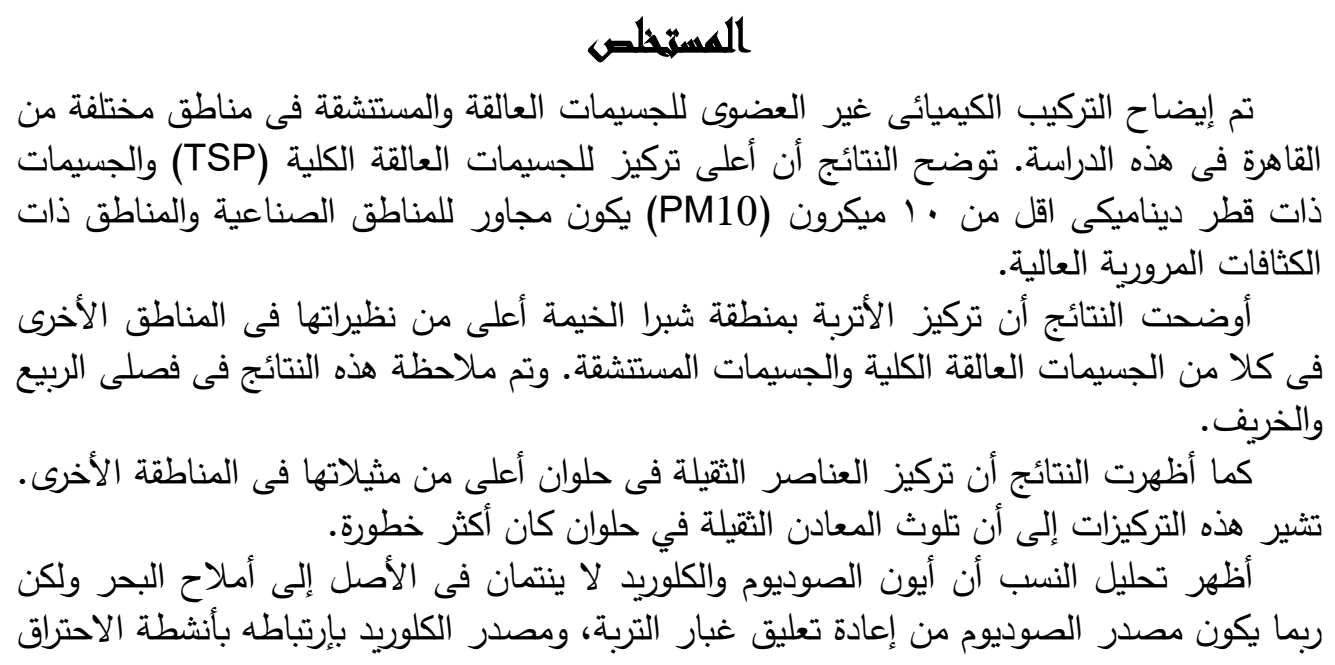

Vol.(50); Iss.(3); No.(1); Mar..2021 
Journal of Environmental Sciences (JES)

Institute of Environmental Studies and Research, Ain Shams University

Elmotily, Hossam et al.

كما أن وجود البوتاسيوم يرجع إلى مصادر متتوعة بخلاف حرق الوقود الحيوى. معظم الكبريتات والنترات ذات أصل الوقود الأحفوري.

أظهرت نسبة أيونات السلفات مع ايوانت النترات النيات فى التبين أكبر من حلوان وشبرا الخيمة فى في

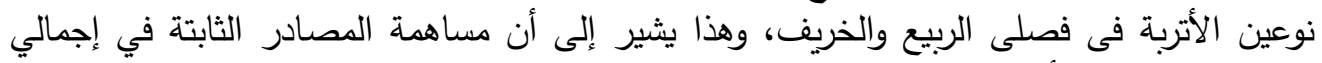

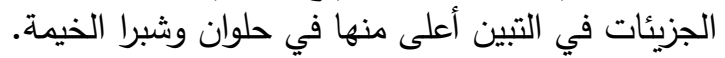

الكلمات الافتتاحية: الجسيمات الدقيقة؛ التركيب الكيميائي غير العضئية العضوي؛ التبين؛ حلوان؛ شبرا الخيمة؛

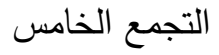

